\title{
Simultaneous Assessment of Transporter-Mediated Drug-Drug Interactions Using a Probe Drug Cocktail in Cynomolgus Monkey
}

\author{
Rachel E. Kosa, Sarah Lazzaro, Yi-an Bi, Brendan Tierney, Dana Gates, Sweta Modi, \\ Chester Costales, A. David Rodrigues, Larry M. Tremaine, and Manthena V. Varma \\ Pharmacokinetics, Dynamics, and Metabolism, Medicine Design (R.E.K., S.L., Y.-a.B., B.T., C.C., A.D.R., L.M.T., M.V.V.) and \\ Research Formulations, Pharmaceutical Sciences (D.G., S.M.), Pfizer Worldwide R\&D, Groton, Connecticut
}

Received April 1, 2018; accepted May 30, 2018

\begin{abstract}
We aim to establish an in vivo preclinical model to enable simultaneous assessment of inhibition potential of an investigational drug on clinically relevant drug transporters, organic anion-transporting polypeptide (OATP)1B, breast cancer resistance protein (BCRP), P-glycoprotein (P-gp), and organic anion transporter (OAT)3. Pharmacokinetics of substrate cocktail consisting of pitavastatin (OATP1B substrate), rosuvastatin (OATP1B/BCRP/OAT3), sulfasalazine (BCRP), and talinolol (P-gp) were obtained in cynomolgus monkey-alone or in combination with transporter inhibitors. Single-dose rifampicin $(30 \mathrm{mg} / \mathrm{kg})$ significantly $(P<0.01)$ increased the plasma exposure of all four drugs, with a marked effect on pitavastatin and rosuvastatin [area under the plasma concentration-time curve (AUC) ratio 21-39]. Elacridar, BCRP/P-gp inhibitor, increased the AUC of sulfasalazine, talinolol, as well as rosuvastatin and pitavastatin. An OAT1/3 inhibitor
\end{abstract}

(probenecid) significantly $(P<0.05)$ impacted the renal clearance of rosuvastatin ( 8-fold). In vitro, rifampicin $(10 \mu \mathrm{M})$ inhibited uptake of pitavastatin, rosuvastatin, and sulfasalazine by monkey and human primary hepatocytes. Transport studies using membrane vesicles suggested that all probe substrates, except talinolol, are transported by cynoBCRP, whereas talinolol is a cynoP-gp substrate. Elacridar and rifampicin inhibited both cynoBCRP and cynoP-gp in vitro, indicating potential for in vivo intestinal efflux inhibition. In conclusion, a probe substrate cocktail was validated to simultaneously evaluate perpetrator impact on multiple clinically relevant transporters using the cynomolgus monkey. The results support the use of the cynomolgus monkey as a model that could enable drug-drug interaction risk assessment, before advancing a new molecular entity into clinical development, as well as providing mechanistic insights on transporter-mediated interactions.

\section{Introduction}

Prediction of drug-drug interaction (DDI) liability of a new molecular entity (NME) is an important factor to consider during drug design/ discovery and development, as such DDIs could impact patient safety or diminish drug efficacy (Bjornsson et al., 2003; Zhang et al., 2007; Bloomer et al., 2013). Transporters of the solute carrier and ATPbinding cassette $(\mathrm{ABC})$ superfamilies are expressed in a variety of organs, including intestine, liver, and kidney, and are increasingly recognized for their key role in the absorption, distribution, clearance, and elimination of drugs (Begley, 2004; Lee and Kim, 2004; Shitara et al., 2006; Koepsell et al., 2007; Feng et al., 2010; Giacomini et al., 2010; Varma et al., 2010; El-Kattan and Varma, 2018). On the basis of established evidences, P-glycoprotein (P-gp), breast cancer resistance protein (BCRP), organic anion-transporting polypeptides (OATPs), organic anion and cation transporters (OATs and OCTs), and multidrug and toxic compound extrusion (MATE) transporters are suggested to be of clinical relevance (Giacomini et al., 2010; Varma et al., 2017a).

All authors are full-time employees of Pfizer. The authors have no conflicts of interest that are directly relevant to this study.

https://doi.org/10.1124/dmd.118.081794.

S This article has supplemental material available at dmd.aspetjournals.org.
Regulatory guidance from agencies, including European Medical Agency, the Pharmaceuticals and Medical Devices Agency (Japan), and United States Food and Drug Administration, now recommends appropriate in vitro and/or in vivo assessment of investigational drugs for potential transporter DDIs (http://www.ema.europa.eu/docs/en_GB/ document_library/Scientific_guideline/2012/2007/WC500129606.pdf; Saito et al., 2014; http://www.fda.gov/downloads/drugs/guidancecomplianceregulatoryinformation/guidances/ucm292362.pdf). For instance, NME with a potential to inhibit OATP1B1, in which in vitro measured inhibition potency $\left(\mathrm{IC}_{50}\right.$ or $\left.\mathrm{Ki}\right) \leq 10$ times the unbound maximum plasma liver inlet concentration at the clinical dose, is predicted to increase systemic exposure of substrate drugs (e.g., statins, sartans, and glinides) (http://www.fda.gov/downloads/drugs/guidancecomplianceregulatoryinformation/guidances/ucm292362.pdf).

Although the unbound maximum plasma liver inlet concentration cannot be physically measured and thus carries some uncertainty, accurate determination of $\mathrm{IC}_{50}$ or $\mathrm{Ki}$ is a key to the reliable prediction using these methods. Intra- and interlaboratory variability in the in vitro $\mathrm{IC}_{50}$ or $\mathrm{Ki}$ values and considerable discrepancy in the in vitro and the estimated in vivo inhibitory potency have been noted previously (Varma et al., 2012; Bentz et al., 2013; Li et al., 2014; Vaidyanathan et al., 2016). For instance, substantial variability was observed in the in vitro P-gp $\mathrm{IC}_{50}$ values of the 16 inhibitors examined at 23 different laboratories

ABBREVIATIONS: ABC, ATP-binding cassette; AUC, area under the plasma concentration-time curve; BCRP, breast cancer resistance protein; $\mathrm{C}_{\max }$, maximum plasma concentration; DDI, drug-drug interaction; ECCS, extended clearance classification system; HBSS, Hanks' balanced salt solution; I I $I_{\text {ut }}$ gut concentration; IS, internal standard; LC-MS/MS, liquid chromatography-tandem mass spectrometry; MATE, multidrug and toxin extrusion protein; NME, new molecular entity; OAT, organic anion transporter; OATP, organic anion-transporting polypeptide; OCT, organic cation transporter; P-gp, P-glycoprotein; $\mathrm{t}_{1 / 2}$, half-life. 
(Bentz et al., 2013). Similarly, we previously noted large interlaboratory variability in the in vitro $\mathrm{IC}_{50}$ values for OATP inhibitors; and the geometric mean of the reported in vitro $\mathrm{Ki}$ values are several folds greater than the estimated apparent in vivo Ki for OATP inhibitors (Varma et al., 2012; Li et al., 2014). Retrospectively, this disconnect may be explained by the time-dependent inhibition (Amundsen et al., 2010; Gertz et al., 2013) or substrate-dependent inhibition (Noé et al., 2007; Izumi et al., 2013) or contribution of the circulating metabolites (e.g., gemfibrozil 1-O- $\beta$-glucuronide) (Shitara et al., 2004; Varma et al., 2015a; Shen et al., 2016). The differences in Ki values with different probe substrates and/or varied experimental settings raise uncertainty in DDI assessment. Overall, compared with cytochrome P450-mediated DDIs, the risk of underestimating potentially hazardous drug interactions involving transporters or transporter-enzyme interplay is relatively high.

Recent clinical studies showed encouraging data regarding the utility of endogenous biomarkers for assessing investigational drugs as an inhibitor, particularly against OATP1B1/1B3, OAT1/3, and OCT2/ MATEs (Lai et al., 2016; Rodrigues et al., 2018). Also, clinical studies employing multiple probe substrate drug cocktails have been proposed to allow simultaneous assessment of inhibition risk (Stopfer et al., 2016; Prueksaritanont et al., 2017). Although these studies show promising results, risk assessment prior to first-in-man studies-needed to nominate the best candidate into clinical development-still remains a major challenge. It is therefore desirable to establish a preclinical animal model that can project the clinical DDI risk. In this regard, some recent studies suggested utility of the cynomolgus monkey to assess inhibition of drug transporters, including OATP, P-gp, BCRP, and OAT1/3 (Tahara et al., 2006; Shen et al., 2013, 2016; Karibe et al., 2015).

In this study, we evaluated a cocktail of probe substrates for early assessment of transporter-mediated DDIs using cynomolgus monkey as a preclinical model. The cocktail consisted of probe substrates of OATPs (pitavastatin and rosuvastatin), P-gp (talinolol), BCRP (sulfasalazine), and OAT3 (rosuvastatin); and validated against a single-dose oral treatment of an OATP inhibitor (rifampicin), P-gp and BCRP inhibitor (elacridar), or OAT1/3 inhibitor (probenecid). In vitro transport (substrate and inhibition) studies were carried out using membrane vesicle systems and primary monkey and human hepatocytes to corroborate the interaction mechanisms.

\section{Materials and Methods}

Chemicals and Reagents. Rifampicin (rifampin) was purchased from SigmaAldrich (St. Louis, MO). Pitavastatin and rosuvastatin were purchased from Sequoia Research Products (Oxford, UK). Probenecid and sulfasalazine were purchased from USP (Rockville, MD), and elacridar and talinolol were purchased from eNovation Chemicals (Bridgewater, NJ). InVitroGro-HT and CP and hepatocyte media were purchased from Celsis IVT (Baltimore, MD). Collagen Icoated 24-well plates were obtained from BD Biosciences (Franklin Lakes, NJ). Cryopreserved cynomolgus monkey hepatocytes (10106012, female) were purchased from In Vitro ADMET Laboratories (Columbia, MD). Cryopreserved human hepatocytes (Hu8246, female, Caucasian, 37 years old) were purchased from Thermo Fisher Scientific (Carlsbad, CA). Bicinchoninic acid protein assay kit was purchased from Pierce (Rockford, IL). Cynomolgus monkey P-gp vesicles (cynoP-gp; gene information, Macaca fascicularis ABCB1, GenBank accession: NM_001287322.1) and BCRP (cynoBCRP; gene information, M. fascicularis ABCG2, GenBank accession: XM_005555388.1) were provided by Solvo Biotechnology (Budapest, Hungary).

In Vivo Studies with Cynomolgus Monkeys. All procedures performed on these animals were in accordance with regulations and established guidelines, were reviewed and approved by Pfizer Institutional Animal Care and Use Committee, and were conducted at Pfizer (Groton, CT). Male cynomolgus macaque Mauritian monkeys (6-9 years of age) were used, and the procedures similar to those previously reported were adopted with some modifications
(Varma et al., 2017b; de Bruyn et al., 2018; Ufuk et al., 2018). A crossover study design was employed, in which the same four animals were dosed over all studies, following a minimum 1-week washout period between each study. Animals were provided a normal food schedule the day before the study (meals at 8:00 AM and 11:00 AM, with one treat daily) and were allowed free access to water. Animals were housed in metabolism cages during sample collection. On the day of the study, monkeys were fed at approximately 1 and 3 hours postdose and allowed water ad libitum. Blank vehicle, rifampicin $(30 \mathrm{mg} / \mathrm{kg})$, elacridar $(30 \mathrm{mg} / \mathrm{kg})$, or probenecid $(30 \mathrm{mg} / \mathrm{kg}$ ) was administered via oral gavage in a $0.5 \% \mathrm{w} / \mathrm{v}$ methylcellulose (in water) suspension 30 minutes prior to oral cassette dosing of pitavastatin $(1 \mathrm{mg} / \mathrm{kg})$, rosuvastatin $(1 \mathrm{mg} / \mathrm{kg})$, talinolol $(1 \mathrm{mg} / \mathrm{kg})$, and sulfasalazine $(0.5 \mathrm{mg} / \mathrm{kg})$. Sulfasalazine (in $2 \% \mathrm{v} / \mathrm{v}$ dimethylsulfoxide: $98 \% \mathrm{v} / \mathrm{v}$ phosphate-buffered saline) and talinolol (in $2 \% \mathrm{v} / \mathrm{v}$ dimethylsulfoxide: $10 \% \mathrm{v} / \mathrm{v}$ polyethylene glycol 400: $88 \% \mathrm{v} / \mathrm{v}$ of $12 \% \mathrm{w} / \mathrm{v}$ sulfobutyl ether $\beta$-cyclodextrin in water) were also dosed individually at 1 and $0.2 \mathrm{mg} / \mathrm{kg}$, respectively, via i.v. administration at a dose volume of $0.5 \mathrm{ml} / \mathrm{kg}$ in separate studies to calculate oral bioavailability. Serial blood samples were collected via the femoral vein into $\mathrm{K}_{2}$ EDTA tubes prior to dosing and then at $0.083,0.25,0.5,0.75,1,2,3,5,6$, and 24 hours postoral cassette dosing.

Blood samples were stored on wet ice prior to being centrifuged to obtain plasma $\left(3000 \mathrm{rpm}, 10\right.$ minutes at $\left.4^{\circ} \mathrm{C}\right)$. Urine was also collected on wet ice, predose and at intervals of $0-6$ and 6-24 hours postdose. Due to the potential instability of rifampicin and possible interconversion of lactone to pitavastatin or rosuvastatin, all plasma and urine samples were equally divided into two aliquots prior to being stored frozen. The first aliquot was untreated matrix, whereas the second aliquot was added to an equal volume of $0.1 \mathrm{M}$ sodium acetate buffer ( $\mathrm{pH} 4$ ). All urine and plasma samples, treated and untreated, were kept cold during collection, after which they were stored frozen at $-20^{\circ} \mathrm{C}$.

Bioanalysis of In Vivo Samples. The concentrations of elacridar, probenecid, rifampicin, talinolol, pitavastatin, rosuvastatin, and sulfasalazine were measured in plasma, and urine samples treated with $0.1 \mathrm{M}$ sodium acetate buffer $(\mathrm{pH} 4)$ using liquid chromatography-tandem mass spectrometry (LC-MS/MS). All standards were prepared in blank monkey plasma mixed with an equal volume of $0.1 \mathrm{M}$ sodium acetate buffer ( $\mathrm{pH}$ 4). Aliquots of $20 \mu \mathrm{l}$ standards or plasma samples were prepared by protein precipitation with $100 \mu \mathrm{l}$ acetonitrile containing an internal standard (IS) mixture of verapamil $(2.5 \mathrm{ng} / \mathrm{ml})$, terfenadine $(30 \mathrm{ng} / \mathrm{ml})$, propranolol $(12.5 \mathrm{ng} / \mathrm{ml})$, and tolbutamide $(5 \mathrm{ng} / \mathrm{ml})$. Urine samples were diluted with nine volumes of a 50/50 mix of blank monkey plasma/ $0.1 \mathrm{M}$ sodium acetate buffer ( $\mathrm{pH} 4)$ and analyzed with the plasma samples. The plates were vortexed for 2 minutes and centrifuged at $3000 \mathrm{rpm}$ for 5 minutes, and $50 \mu 1$ supernatants of the mixture were transferred to a new sample block, diluted with $100 \mu \mathrm{l}$ water containing $0.1 \%$ formic acid, and analyzed via LC-MS/MS

Chromatography was performed on a Waters Acquity iClass UPLC System (Milford, MA). The autosampler and column were kept at $10^{\circ} \mathrm{C}$ and $40^{\circ} \mathrm{C}$, respectively. Separation was achieved with an Acquity UPLC HSS T3 column $(2.1 \times 50 \mathrm{~mm}, 1.8 \mu \mathrm{m}$ ) and a gradient of $0.1 \%$ formic acid in water (mobile phase A) and $0.1 \%$ formic acid in acetonitrile (mobile phase B) at a flow rate of $0.600 \mathrm{ml} / \mathrm{min}$. An initial mobile-phase composition of $5 \% \mathrm{~B}$ was held for 0.3 minute, ramped to $95 \%$ over 2 minutes, held at $95 \%$ for 0.3 minute, and then returned to initial $5 \% \mathrm{~B}$ over 0.1 minute for re-equilibration. A Valco VICI valve (Valco Instruments, Houston, TX) was used to divert the first 0.3 minute and the last 0.3 minute of high-performance liquid chromatography effluent to waste. The total run time for each injection was 3 minutes. Data were collected on an $\mathrm{AB}$ Sciex API5500 mass spectrometer (Applied Biosystems, Foster City, CA) using either positive or negative Turbo IonSpray electrospray ionization and multiple reaction monitoring mode. Typical source conditions (heated capillary temperature, gas1, gas 2 , and curtain gas) were set at $500^{\circ} \mathrm{C}, 40^{\circ} \mathrm{C}, 60^{\circ} \mathrm{C}$, and $20^{\circ} \mathrm{C}$, respectively. Multiple reaction monitoring transitions for each analyte, along with the IS used, are included in a Supplemental Table 1.

Uptake Studies Using Cryopreserved Plateable Monkey Hepatocytes (PMH). The hepatic uptake assay was performed using short-term culture format, as described previously, with some modification (Bi et al., 2017, 2018). Briefly, cryopreserved cynomolgus hepatocytes and human hepatocytes were thawed in the InVitro-HT media at $37^{\circ} \mathrm{C}$ at a ratio of one or two vials $/ 50 \mathrm{ml}$ in a conical tube. The cells were centrifuged at $50 \mathrm{~g}$ for 3 minutes and resuspended in In VitroGro-CP medium. The cells were seeded into 24-well collagen I-coated plates with $0.35 \times 10^{6}$ cells/well in a volume of $0.5 \mathrm{ml} / \mathrm{well}$. The cells were cultured in the InVitro-CP media overnight ( $\sim 18$ hours). Cell culture was first 
rinsed twice with Hanks' balanced salt solution (HBSS) buffer and then preincubated for 30 minutes at $37^{\circ} \mathrm{C}$ with HBSS with or without inhibitors. The preincubation buffer was aspirated, and the uptake and inhibition reaction were initiated by addition of prewarmed buffer containing substrates with or without inhibitors. The reactions were terminated at designated time points $(0.5$ and 2 minutes) by adding ice-cold HBSS immediately after removal of the incubation buffer. The cells were washed three times with ice-cold HBSS and lysed with $100 \%$ methanol containing IS, and the samples were analyzed by LC-MS/MS (Supplemental Material). Uptake rates were estimated from the initial time course (0.5-2 minutes) by linear regression.

In Vitro Transport Studies Using Membrane Vesicles. Vesicle assay buffer (10 mM Tris base, $250 \mathrm{mM}$ sucrose, $10 \mathrm{mM}$ magnesium chloride) and stop buffer (assay buffer plus $100 \mathrm{mM}$ sodium chloride) were prepared at $\mathrm{pH}$ 7.4. For substrate assays, HEK293-cynoBCRP, HEK293-cynoP-gp, and HEK293-mock vesicles were diluted to $50 \mu \mathrm{g} / \mathrm{well}$ in assay buffer and treated with $5 \mathrm{mM}$ ATP and $1 \mu \mathrm{M}$ test compound (pitavastatin, rosuvastatin, sulfasalazine, talinolol, or $\mathrm{n}$-methyl quinidine). Assays were incubated at $22^{\circ} \mathrm{C}$ while shaking. Reactions were stopped at $0.25,0.5,1,2.5,5$, and 10 minutes by the addition of $200 \mu 1$ icecold stop buffer. The entire reaction was quickly removed from the assay plate and filtered on a vacuum filter plate (Multiscreen; Millipore Burlington, MA) and then washed four times with ice-cold stop buffer. For inhibition assays, HEK293cynoBcrp and HEK293-cynoP-gp vesicles were diluted to $50 \mu \mathrm{g} / \mathrm{well}$ in assay buffer and were treated with $5 \mathrm{mM}$ ATP, $0.2 \mu \mathrm{M}$ rosuvastatin (BCRP), or $0.2 \mu \mathrm{M}$ n-methyl quinidine (P-gp) and varied concentrations of inhibitor (elacridar, probenecid, rifampicin, or control inhibitors Ko143 and PCS833). BCRP and P-gp assays were incubated for 1 and 2 minutes, respectively, at $22^{\circ} \mathrm{C}$, while shaking, and were stopped, as described above.

Intravesicle samples for BCRP and P-gp incubations were extracted by treating the vesicles with $0.1 \mathrm{ml}$ IS solution in $100 \%$ methanol. Vesicles were shaken for 15 minutes at room temperature. Vesicle extracts were transferred to 96-well polypropylene deep-well plates by centrifugation and dried down under nitrogen. Samples were reconstituted in 50:50 methanol:water prior to injection onto a LC-MS/MS system. Samples were analyzed for probe and IS peak areas. LC-MS/MS methods are described in Supplemental Material.

Data for BCRP and P-gp assays were acquired using Analyst 1.6.2, and peak area ratios (analyte:IS) were exported into Microsoft Excel. For substrate assays,
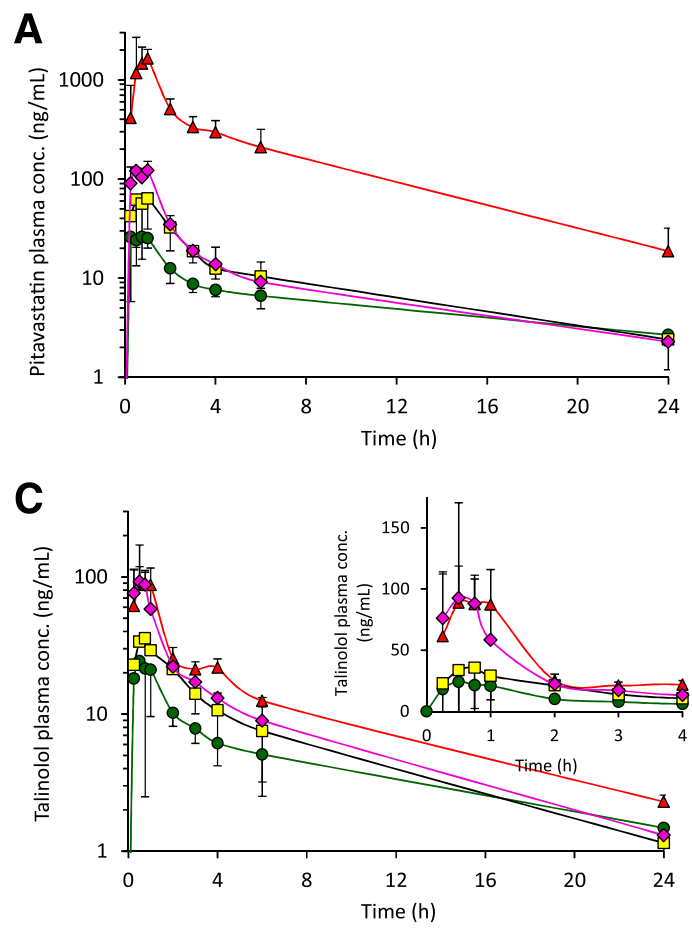

the amount of analyte uptake was calculated as transfected:mock peak area ratio. For inhibition assays, percent transporter activity was calculated by the following equation:

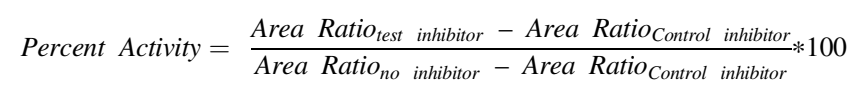

Percent activity values were exported into GraphPad Prism to estimate $\mathrm{IC}_{50}$ values.

Pharmacokinetic Analysis and Statistics. LC-MS/MS data acquisition and peak integration of plasma and urine samples were performed using Analyst 1.6.2 software (Applied Biosystems/MDS Sciex, Ontario, Canada). Peak area values were imported into Watson LIMS version 7.5 (Thermo Fisher Scientific, Waltham, MA) for standard curve regression, quantitation, and for calculation of model independent, noncompartmental pharmacokinetic parameters: area under the plasma concentration-time curve (AUC), maximum plasma concentration $\left(\mathrm{C}_{\mathrm{max}}\right)$, and half-life $\left(\mathrm{t}_{1 / 2}\right)$. One-way analysis of variance was applied to test statistical significance across groups with Tukey's test for multiple comparisons (GraphPad Software, San Diego, CA).

\section{Results}

Effect of Inhibitors on the Oral Pharmacokinetics of Probe Substrates in Cynomolgus Monkey. Oral pharmacokinetics of four probe substrate drugs (pitavastatin, rosuvastatin, talinolol, and sulfasalazine) in a cocktail dosed after treatment with vehicle control or inhibitor drugs (rifampicin, elacridar, probenecid) were studied in cynomolgus monkeys $(n=4)$ (Fig. 1). Dose-normalized pharmacokinetics of the probe drugs obtained following cocktail dose in the control arm of this study are in good agreement with the data reported following individual oral dosing (Karibe et al., 2015, 2018; Shen et al., 2015; Varma et al., 2017). Single-dose oral rifampicin (30 mg/kg) significantly (one-way analysis of variance, $P<0.01$ ) increased the plasma exposure of all four probe drugs (Fig. 2; Tables 1-4). Particularly, rifampicin showed marked effects on OATP substrates, pitavastatin and
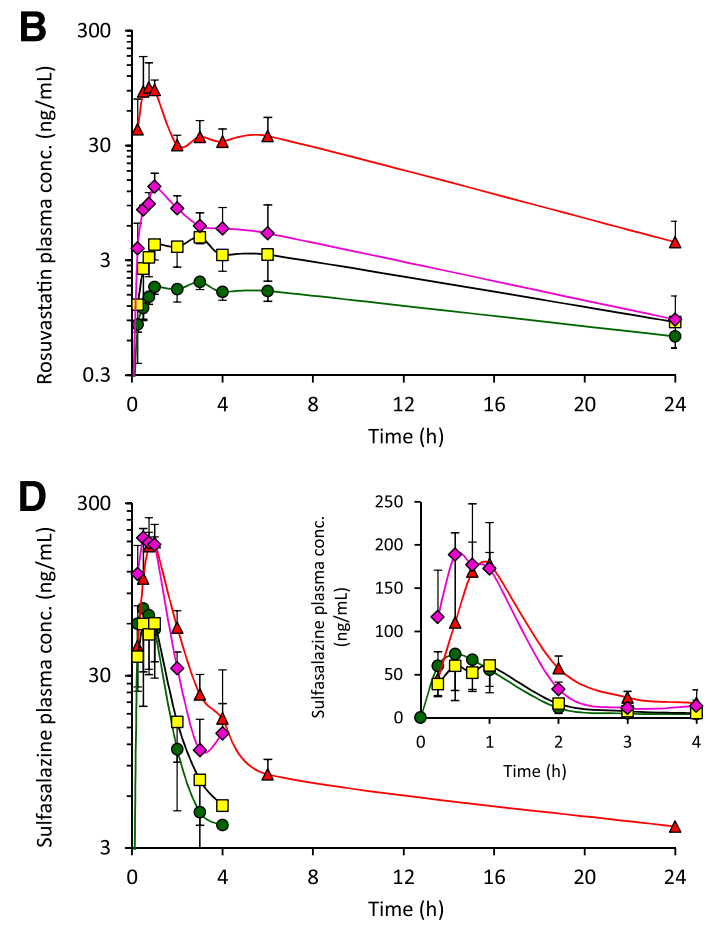

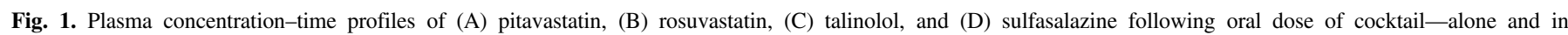

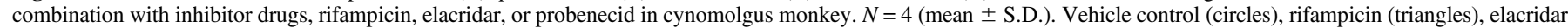
(diamonds), or probenecid (squares) was dosed 1 hour prior to the oral dose of probe drugs cassette. Inserts show early time points on linear scale for clarity. 

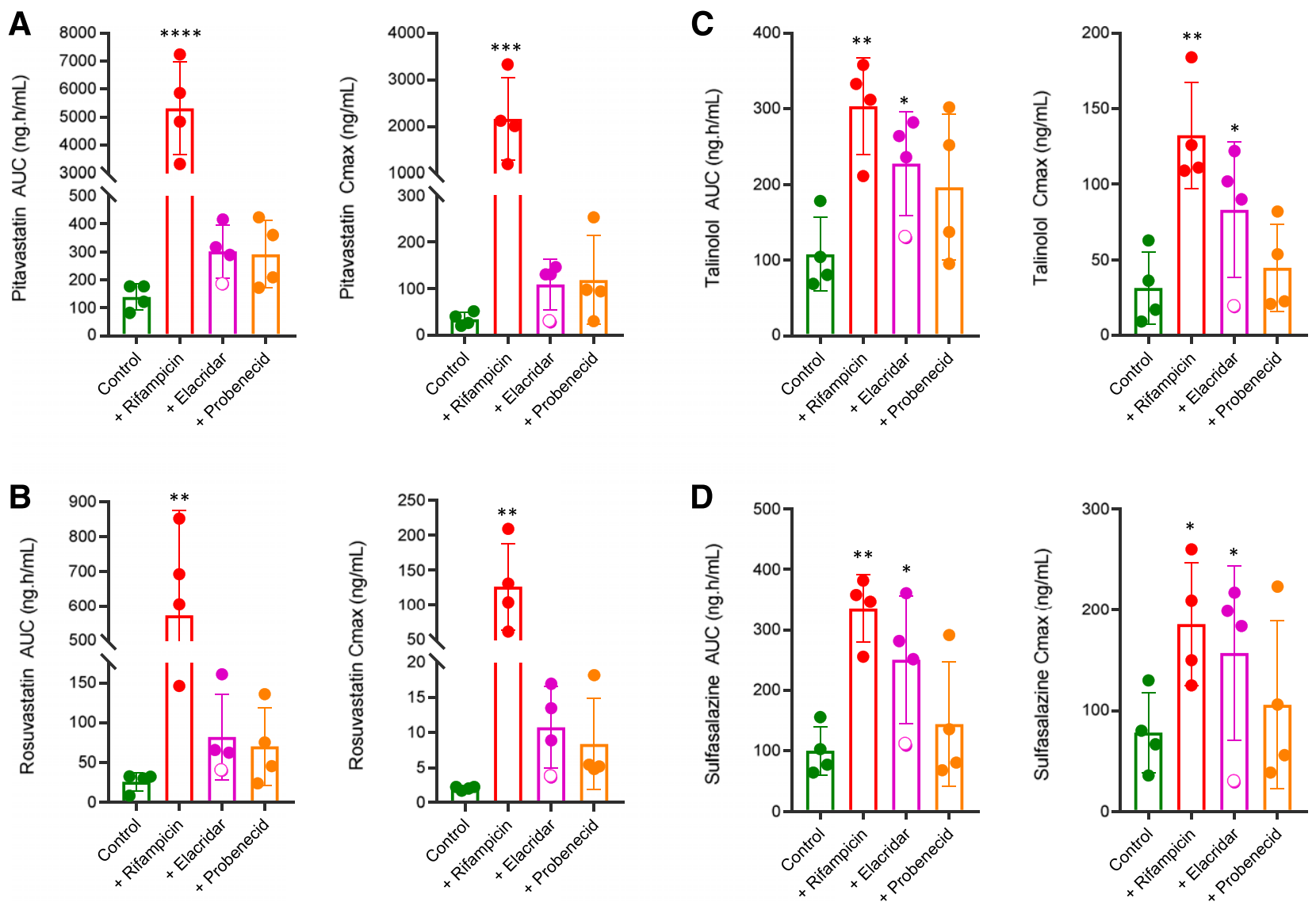

Fig. 2. Effect of single-dose rifampicin, elacridar, and probenecid $\left(30 \mathrm{mg} / \mathrm{kg}\right.$ each) on the plasma $\mathrm{AUC}_{0-\mathrm{t}}$ and $\mathrm{C}_{\max }$ of transporter probe drugs (A) pitavastatin, (B) rosuvastatin, (C) talinolol, and (D) sulfasalazine following oral dosing of cocktail in cynomolgus monkey. Bars and error bars represent mean \pm S.D. $(n=4)$. Open data point, excluded for the analysis in elacridar treatment group due to considerable deviation from the remaining three animals. Individual data points also shown. $* * * * P<$ $0.0001 ; * * P<0.001 ; * * P<0.01 ; * P<0.05$ (one-way analysis of variance with Tukey test for multiple group comparison).

rosuvastatin-with AUC ratios (ratio of AUC in the presence of inhibitor to vehicle control) of about $39 \pm 22$ (geometric mean \pm S.D.) and $21 \pm 35$, respectively. Interindividual variability in AUC was relatively high $(\mathrm{CV} \sim 50 \%)$ for rosuvastatin in each arm, resulting in $\%$ CVs up to $100 \%$ in AUC ratios ( \pm rifampicin). Variability in AUC of other probes is generally low $(25 \%-40 \% \mathrm{CV})$.

Rifampicin also significantly increased the plasma $\mathrm{C}_{\max }$ of all probe drugs (Fig. 2; Tables 1-4). P-gp and BCRP inhibitor, elacridar, significantly increased plasma AUC of talinolol and sulfasalazine. Although statistical significance was not reached $(P>0.05)$, the plasma exposure of pitavastatin and rosuvastatin was increased by $\sim 2.8$ - and 4.4-fold, respectively, with elacridar. Probenecid, an OAT1/3 inhibitor, did not present a significant effect on the plasma exposure of all the drugs tested, although an increasing trend was apparent for pitavastatin and rosuvastatin ( $\sim$ twofold). No significant change $(P>0.05)$ in the terminal $t_{1 / 2}$ of the probe drugs was observed. This reflects expected decrease in both systemic clearance and volume of distribution of statins due to OATP1B inhibition by rifampicin (Ufuk et al., 2018). For talinolol and sulfasalazine, lack of $t_{1 / 2}$ change may suggest limited effect of the inhibitors on their systemic clearance. The time at maximum plasma concentration $\left(\mathrm{T}_{\max }\right.$ ) of pitavastatin, talinolol, and sulfasalazine was not affected by any inhibitor. However, rifampicin and elacridar reduced the $\mathrm{T}_{\max }$ of rosuvastatin from about 3.5 to $0.8-1.8$ hours.

Probenecid, but not rifampicin or elacridar, markedly decreased $(P<$ $0.05)$ the renal clearance of rosuvastatin-renal clearance ratio (i.e., $\mathrm{CL}_{\text {renal,+inh }} / \mathrm{CL}_{\text {renal,control }}$ ) of $\sim 7.8$ (Table 2 ). However, talinolol renal clearance was not inhibited by any inhibitor. In contrast, urinary excretion of pitavastatin and sulfasalazine is negligible or often undetectable.

Plasma samples from these studies were measured to obtain concentration-time profiles of inhibitor drugs (Fig. 3). The unbound $\mathrm{C}_{\max }$ of rifampicin was about $10.7 \mu \mathrm{M}$ (total $\mathrm{C}_{\max }, 50.8 \mu \mathrm{M}$; measured monkey $\left.\mathrm{f}_{\mathrm{u} \text {,plasma }}, 0.21\right)$. In the case of elacridar, unbound $\mathrm{C}_{\max }$ is less than $0.1 \mathrm{nM}$ (total $\mathrm{C}_{\max }, \sim 100 \mathrm{nM} ; \mathrm{f}_{\mathrm{u} \text {,plasma }}, 0.001$ ). In contrast, unbound $\mathrm{C}_{\max }$ of probenecid at this given dose is about $76 \mu \mathrm{M}$ (total $\left.\mathrm{C}_{\max }, 588 \mu \mathrm{M} ; \mathrm{f}_{\mathrm{u} \text {,plasma }}, 0.13\right)$. Through the time course of 24 hours, rifampicin plasma-unbound concentrations are well above the in vitro $\mathrm{IC}_{50}(0.54 \mu \mathrm{M})$ (Ufuk et al., 2018) for uptake inhibition in monkey hepatocytes (Fig. 3). Similarly, probenecid plasma-unbound concentrations are several fold higher than its in vitro $\mathrm{IC}_{50}(3.0 \mu \mathrm{M})$ (Tahara et al., 2006) obtained in monkey OAT3-transfected cells. Monkey plasmaunbound concentrations of elacridar are, however, negligible. Additionally, unbound concentrations reached in the present monkey studies are within the range observed in humans: $2-6 \mu \mathrm{M}$ for $600 \mathrm{mg}$ rifampicin (Varma et al., 2012; Prueksaritanont et al., 2014; Yoshikado et al., 2017); <1 nM for $1000 \mathrm{mg}$ elacridar (Sawicki et al., 2017); and 6-51 $\mu \mathrm{M}$ for $500-1500 \mathrm{mg}$ probenecid (Mathialagan et al., 2017).

Effect of Inhibitors on the In Vitro Uptake of Probe Substrates by Cynomolgus Monkey and Human Hepatocytes. Uptake of each of the four probe substrate drugs was evaluated in monkey and human hepatocytes (short-term culture format) in the absence and presence of the inhibitors-at concentrations close to their corresponding unbound $\mathrm{C}_{\max }$ for rifampicin and probenecid. Elacridar was incubated at $0.1 \mu \mathrm{M}$, which is much above its unbound $\mathrm{C}_{\max }$. Rifampicin $(10 \mu \mathrm{M})$ 
TABLE 1

Summary of pharmacokinetic parameters of pitavastatin following oral dosing of probe drug cassette in cynomolgus monkey, alone or in combination with single oral doses of rifampicin, elacridar, or probenecid

Values depict mean \pm S.D. for pharmacokinetic $(P K)$ parameters and (geometric mean \pm S.D.) where ratio of treatment to control group was shown. Statistical significance for $A U C_{0-t}, C_{\max }, t_{1 / 2}$, and $C L_{\text {renal }}$ was tested by one-way analysis of variance with Tukey test for multiple group comparison.

\begin{tabular}{|c|c|c|c|c|}
\hline PK Parameters & Control & + Rifampicin & + Elacridar $^{a}$ & + Probenecid \\
\hline $\mathrm{AUC}_{0-\mathrm{t}}(\mathrm{ng} \times \mathrm{h} / \mathrm{ml})$ & $139 \pm 46$ & $5322 \pm 1655^{* * * * *}$ & $341 \pm 67$ & $291 \pm 120$ \\
\hline AUC ratio & - & $(39 \pm 22)$ & $(2.8 \pm 1.0)$ & $(2.1 \pm 1.0)$ \\
\hline $\operatorname{AUC}_{0-\text { inf }}(\mathrm{ng} \times \mathrm{h} / \mathrm{ml})$ & $206 \pm 25$ & $5460 \pm 1750$ & $372 \pm 54$ & $325 \pm 106$ \\
\hline $\mathrm{C}_{\max }(\mathrm{ng} / \mathrm{ml})$ & $35 \pm 14$ & $2162 \pm 882 * * *$ & $136 \pm 9.2$ & $119 \pm 95$ \\
\hline $\mathrm{C}_{\max }$ ratio & - & $(62 \pm 33)$ & $(3.9 \pm 2.6)$ & $(2.8 \pm 5.3)$ \\
\hline $\mathrm{T}_{\max }(\mathrm{h})$ & $0.7 \pm 0.3$ & $0.8 \pm 0.2$ & $0.6 \pm 0.4$ & $0.9 \pm 0.3$ \\
\hline$t_{1 / 2}(h)$ & $13.9 \pm 5.9$ & $4.9 \pm 0.5$ & $9.4 \pm 3.0$ & $6.9 \pm 3.2$ \\
\hline$\%$ Bioavailability ${ }^{b}$ & $20 \pm 5$ & - & - & - \\
\hline$\%$ Unchanged drug in urine in $24 \mathrm{~h}$ & $0.3 \pm 0.2$ & $5.3 \pm 1.4$ & $0.2 \pm 0.2$ & ND \\
\hline $\mathrm{CL}_{\mathrm{renal}}(\mathrm{ml} / \mathrm{min}$ per kilogram $)$ & $0.2 \pm 0.1$ & $0.2 \pm 0.1$ & $0.2 \pm 0.1$ & ND \\
\hline
\end{tabular}

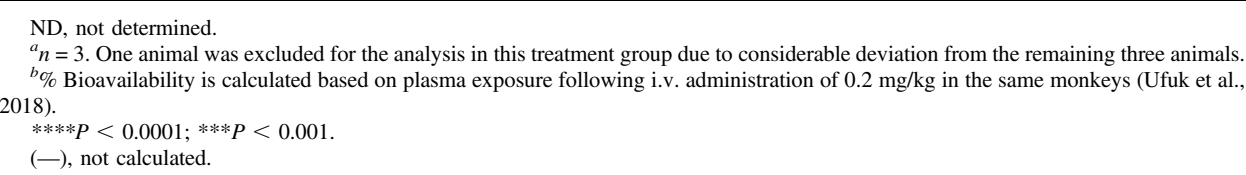

significantly inhibited uptake of pitavastatin, rosuvastatin, and sulfasalazine, in both monkey and human hepatocytes (Fig. 4). Elacridar $(0.1 \mu \mathrm{M})$ did not show inhibition of hepatic uptake for any substrate. Interestingly, probenecid $(50 \mu \mathrm{M})$ significantly inhibited uptake of statins and sulfasalazine by cynomolgus monkey hepatocytes; however, only a marginal inhibition (10\%-15\%) was noted in human hepatocytes. Apart from this difference, interaction mechanism(s) involving hepatic uptake for each substrate-inhibitor pair is comparable across the two species.

Effect of Inhibitors on the In Vitro Transport of Probe Substrates in cynoBCRP and cynoP-gp Membrane Vesicles. We first evaluated pitavastatin, rosuvastatin, sulfasalazine, and talinolol for their substrate affinity to cynoBCRP and cynoP-gp using membrane vesicles prepared from transfected HEK293 cells (Fig. 5). Timedependent uptake was observed with cynoBCRP vesicles for pitavastatin, rosuvastatin, and sulfasalazine. Talinolol showed significant uptake by cynoP-gp vesicles compared with mock vesicles. Interestingly, P-gp-mediated transport was also seen for rosuvastatin, although the activity is notably small compared with cynoBCRP activity (Fig. 5, $\mathrm{B}$ and $\mathrm{F})$.
Using rosuvastatin and N-methyl-quinidine (NMQ) as substrates (validated for the intended use with control inhibitors Ko143 and PSC833, respectively), cynoBCRP and cynoP-gp inhibition was evaluated for elacridar, rifampicin, and probenecid. Elacridar and rifampicin showed concentration-dependent inhibition of both cynoBCRP- and cynoP-gp-mediated transport, whereas probenecid had a minimal effect up to $300 \mu \mathrm{M}$ (Fig. 6). Inhibition potency $\left(\mathrm{IC}_{50}\right)$ estimates are provided in Table 5. Additionally, $\mathrm{IC}_{50}$ of the inhibitor drugs against human BCRP and P-gp were extracted from the literature and compared with the cynomolgus monkey. Based on the ratio of gut concentration $\left(\mathrm{I}_{\mathrm{Gut}}\right.$; calculated as a ratio of dose to gut volume, in which gut volume was assumed to be $250 \mathrm{ml}$ for humans and $10 \mathrm{ml} / \mathrm{kg}$ for monkey) (Amidon et al., 1995; Kararli, 1995) to $\mathrm{IC}_{50}$, elacridar and rifampicin show high potential for in vivo intestinal efflux inhibition in both cynomolgus monkey and human (i.e., $\mathrm{I}_{\mathrm{Gut}} / \mathrm{IC}_{50}>>10$ ) (Table 5).

\section{Discussion}

De-risking DDIs involving membrane transporters is an essential part of medicinal chemistry design and drug development. However,

TABLE 2

Summary of pharmacokinetic parameters of rosuvastatin following oral dosing of probe drug cassette in cynomolgus monkey, alone or in combination with single oral doses of rifampicin, elacridar, or probenecid

Values depict mean \pm S.D. for pharmacokinetic $(P K)$ parameters and (geometric mean \pm S.D.) where ratio of treatment to control group was shown. Statistical significance for $A U C_{0-t}, C_{\max }, t_{1 / 2}$, and $C L_{\text {renal }}$ was tested by one-way analysis of variance with Tukey test for multiple group comparison.

\begin{tabular}{|c|c|c|c|c|}
\hline PK Parameters & Control & + Rifampicin & + Elacridar $^{a}$ & + Probenecid \\
\hline $\mathrm{AUC}_{0-\mathrm{t}}(\mathrm{ng} \times \mathrm{h} / \mathrm{ml})$ & $26 \pm 12$ & $573 \pm 302 * *$ & $96 \pm 56$ & $70 \pm 49$ \\
\hline AUC ratio & - & $(21.3 \pm 35)$ & $(4.4 \pm 3.1)$ & $(2.6 \pm 1.3)$ \\
\hline $\operatorname{AUC}_{0-\text { inf }}(\mathrm{ng} \times \mathrm{h} / \mathrm{ml})$ & $41 \pm 9$ & $682 \pm 180$ & $106 \pm 61$ & $92 \pm 33$ \\
\hline $\mathrm{C}_{\max }(\mathrm{ng} / \mathrm{ml})$ & $2.0 \pm 0.3$ & $127 \pm 62 * *$ & $13.1 \pm 4.1$ & $8.4 \pm 6.5$ \\
\hline $\mathrm{C}_{\max }$ ratio & - & $(57 \pm 42)$ & $(6.5 \pm 1.2)$ & $(3.5 \pm 2.8)$ \\
\hline $\mathrm{T}_{\max }(\mathrm{h})$ & $3.5 \pm 1.7$ & $0.8 \pm 0.2$ & $1.8 \pm 1.5$ & $3.0 \pm 2.2$ \\
\hline$t_{1 / 2}(h)$ & $13.0 \pm 3.8$ & $6.9 \pm 3.1$ & $7.6 \pm 0.9$ & $10.5 \pm 7.5$ \\
\hline$\%$ Bioavailability ${ }^{b}$ & $6 \pm 3$ & - & - & - \\
\hline$\%$ Unchanged drug in urine in $24 \mathrm{~h}$ & $1.1 \pm 0.4$ & $9.3 \pm 3.3$ & $1.3 \pm 0.8$ & $0.3 \pm 0.1$ \\
\hline $\mathrm{CL}_{\text {renal }}(\mathrm{ml} / \mathrm{min}$ per kilogram) & $5.3 \pm 1.9$ & $3.3 \pm 1.7$ & $3.6 \pm 2.8$ & $1.0 \pm 0.7 *$ \\
\hline $\mathrm{CL}_{\text {renal }}$ ratio & & $(1.4 \pm 1.1)$ & $(2.1 \pm 1.4)$ & $(7.8 \pm 3.7)$ \\
\hline
\end{tabular}

${ }^{a} n=3$. One animal was excluded for the analysis in this treatment group due to considerable deviation from the remaining three animals 2018 )

$* * P<0.01 ; * P<0.05$.

(一), not calculated. 
TABLE 3

Summary of pharmacokinetic parameters of a P-gp probe substrate, talinolol, following oral dosing of probe drug cassette in cynomolgus monkey, alone or in combination with single oral doses of rifampicin, elacridar, or probenecid

Values depict mean \pm S.D. for pharmacokinetic $(P K)$ parameters and (geometric mean \pm S.D.) where ratio of treatment to control group was shown. Statistical significance for $A U C_{0-t}, C_{\text {max }} t_{1 / 2}$, and $C L_{\text {renal }}$ was tested by one-way analysis of variance with Tukey test for multiple group comparison.

\begin{tabular}{|c|c|c|c|c|}
\hline PK Parameters & Control & + Rifampicin & + Elacridar $^{a}$ & + Probenecid \\
\hline $\mathrm{AUC}_{0-\mathrm{t}}(\mathrm{ng} \times \mathrm{h} / \mathrm{ml})$ & $108 \pm 49$ & $304 \pm 65^{* *}$ & $261 \pm 23 *$ & $196 \pm 97$ \\
\hline AUC ratio & - & $(3.0 \pm 0.9)$ & $(2.4 \pm 1.0)$ & $(1.8 \pm 0.4)$ \\
\hline $\operatorname{AUC}_{0-\text { inf }}(\mathrm{ng} \times \mathrm{h} / \mathrm{ml})$ & $131 \pm 44$ & $335 \pm 46$ & $270 \pm 22$ & $208 \pm 94$ \\
\hline $\mathrm{C}_{\max }(\mathrm{ng} / \mathrm{ml})$ & $31 \pm 24$ & $133 \pm 35^{* *}$ & $105 \pm 16^{*}$ & $45 \pm 29$ \\
\hline $\mathrm{C}_{\max }$ ratio & - & $(5.3 \pm 4.2)$ & $(3.1 \pm 1.7)$ & $(1.5 \pm 1.1)$ \\
\hline $\mathrm{T}_{\max }(\mathrm{h})$ & $1.1 \pm 0.6$ & $0.8 \pm 0.3$ & $0.5 \pm 0.2$ & $1.1 \pm 0.6$ \\
\hline $\mathrm{t}_{1 / 2}(\mathrm{~h})$ & $8.8 \pm 3.8$ & $5.7 \pm 1.4$ & $5.0 \pm 1.8$ & $6.6 \pm 2.1$ \\
\hline$\%$ Bioavailability ${ }^{b}$ & $9 \pm 2$ & - & - & - \\
\hline$\%$ Unchanged drug in urine in $24 \mathrm{~h}$ & $2.2 \pm 0.7$ & $2.4 \pm 0.7$ & $1.8 \pm 1.3$ & $1.1 \pm 0.5$ \\
\hline $\mathrm{CL}_{\text {renal }}(\mathrm{ml} / \mathrm{min}$ per kilogram $)$ & $2.9 \pm 1.2$ & $1.3 \pm 0.4$ & $0.8 \pm 0.4$ & $1.1 \pm 0.5$ \\
\hline $\mathrm{CL}_{\text {renal }}$ ratio & & $(2.2 \pm 1.0)$ & $(2.8 \pm 0.8)$ & $(2.6 \pm 0.8)$ \\
\hline
\end{tabular}

${ }^{a} n=3$. One animal was excluded for the analysis in this treatment group due to considerable deviation from the remaining three animals

${ }^{b} \%$ Bioavailability is calculated based on plasma exposure following single i.v. administration of $0.2 \mathrm{mg} / \mathrm{kg}$ in the same monkeys (data not shown).

$* * P<0.01 ; * P<0.05$.

$(-)$, not calculated.

translation of in vitro $\mathrm{IC}_{50}$ (or $\mathrm{Ki}$ ) values to assess DDI potential is challenged with poorly understood substrate-, incubation time-, and in vitro system-dependent measurements. In this study, we evaluated a cocktail of probe substrates to simultaneously assess the in vivo inhibition potency of NMEs against clinically relevant transporters, OATP1B1/1B3, P-gp, BCRP, and OAT3, using cynomolgus monkey as a preclinical animal model. The proposed cocktail of four probe drugs has been validated in vivo using relatively selective inhibitor drugs. In vitro studies using primary hepatocytes and membrane vesicles verified the interaction mechanisms in the cynomolgus monkey, and further implied similarities in the transporter-mediated disposition in this preclinical model and humans. Although the concept of a transporter probe drug cocktail has been tested clinically (Stopfer et al., 2016; Prueksaritanont et al., 2017), to our knowledge this is the first report of a study employing such a cocktail in cynomolgus monkeys. This approach can thus be valuable in assessing the transporter inhibition potential of NMEs before advancing into first-in-man studies, in rational staging of clinical DDI studies during drug development, and to substantiate in vitro findings in the regulatory submissions.
The probe substrates selected for the proposed cocktail are shown to be associated with the drug transporters of interest based on the clinical pharmacokinetics, DDI, and pharmacogenomics studies. Pitavastatin and rosuvastatin represent extended clearance classification system (ECCS) class 1B and class 3B, respectively, in which OATP-mediated hepatic uptake plays a rate-determining role in the systemic clearance in humans (Shitara et al., 2013; Varma et al., 2015b; El-Kattan et al., 2016). In vitro, both statins are transported by human OATP1B1/1B3/2B 1 and $\mathrm{Na}^{+}$-taurocholate cotransporting polypeptide (NTCP), whereas rosuvastatin is also a substrate to BCRP (Shitara et al., 2013). Renal clearance of pitavastatin in humans is low, but rosuvastatin shows up to $30 \%$ dose excretion in urine and involves OAT3-mediated renal secretion (Mathialagan et al., 2017). OATP1B1/1B3 inhibition by a single-dose rifampicin perpetrates $\sim 3$ - to sevenfold AUC increase for rosuvastatin and pitavastatin in humans (Prueksaritanont et al., 2014, 2017). In contrast, sulfasalazine (ECCS class 3A) and talinolol (ECCS class 4) are low permeability drugs with limited oral absorption in humans (El-Kattan et al., 2016). Consequently, clinical pharmacokinetics of sulfasalazine and talinolol are considerably altered when

TABLE 4

Summary of pharmacokinetic parameters of a BCRP probe substrate, sulfasalazine, following oral dosing of probe drug cassette in cynomolgus monkey, alone or in combination with single oral doses of rifampicin, elacridar, or probenecid

Values depict mean $\pm S . D$. for pharmacokinetic $(P K)$ parameters and (geometric mean \pm S.D.) where ratio of treatment to control group was shown. Statistical significance for $A U C_{0-t}, C_{\max }, t_{1 / 2}$, and $C L_{\text {renal }}$ was tested by one-way analysis of variance with Tukey test for multiple group comparison.

\begin{tabular}{|c|c|c|c|c|}
\hline PK Parameters & Control & + Rifampicin & + Elacridar $^{a}$ & + Probenecid \\
\hline $\mathrm{AUC}_{0-\mathrm{t}}(\mathrm{ng} \times \mathrm{h} / \mathrm{ml})$ & $100 \pm 41$ & $336 \pm 55^{* *}$ & $298 \pm 56^{*}$ & $144 \pm 102$ \\
\hline AUC ratio & - & $(3.5 \pm 1.3)$ & $(3.2 \pm 1.9)$ & $(1.3 \pm 1.8)$ \\
\hline $\operatorname{AUC}_{0-\text { inf }}(\mathrm{ng} \times \mathrm{h} / \mathrm{ml})$ & $104 \pm 38$ & $364 \pm 61$ & $397 \pm 220$ & $150 \pm 103$ \\
\hline $\mathrm{C}_{\max }(\mathrm{ng} / \mathrm{ml})$ & $78 \pm 39$ & $186 \pm 61 *$ & $200 \pm 17 *$ & $106 \pm 83$ \\
\hline $\mathrm{C}_{\max }$ ratio & - & $(2.5 \pm 2.0)$ & $(2.8 \pm 2.1)$ & $(1.2 \pm 2.8)$ \\
\hline $\mathrm{T}_{\max }(\mathrm{h})$ & $0.6 \pm 0.3$ & $0.9 \pm 0.1$ & $0.7 \pm 1.4$ & $0.9 \pm 0.3$ \\
\hline $\mathrm{t}_{1 / 2}(\mathrm{~h})$ & $0.7 \pm 0.5$ & $4.0 \pm 5.0$ & $3.6 \pm 5.1$ & $0.7 \pm 0.3$ \\
\hline$\%$ Bioavailability ${ }^{b}$ & $5 \pm 1$ & - & - & - \\
\hline$\%$ Unchanged drug in urine in $24 \mathrm{~h}$ & ND & $1.3 \pm 0.4$ & $1.4 \pm 0.2$ & ND \\
\hline $\mathrm{CL}_{\text {renal }}(\mathrm{ml} / \mathrm{min}$ per kilogram) & ND & $0.3 \pm 0.1$ & $0.4 \pm 0.1$ & ND \\
\hline
\end{tabular}

$\mathrm{ND}$, not determined.

${ }_{n} n=3$. One animal was excluded for the analysis in this treatment group due to considerable deviation from the remaining three animals. 2015).

$* * P<0.01 ; * P<0.05$

(一), not calculated. 

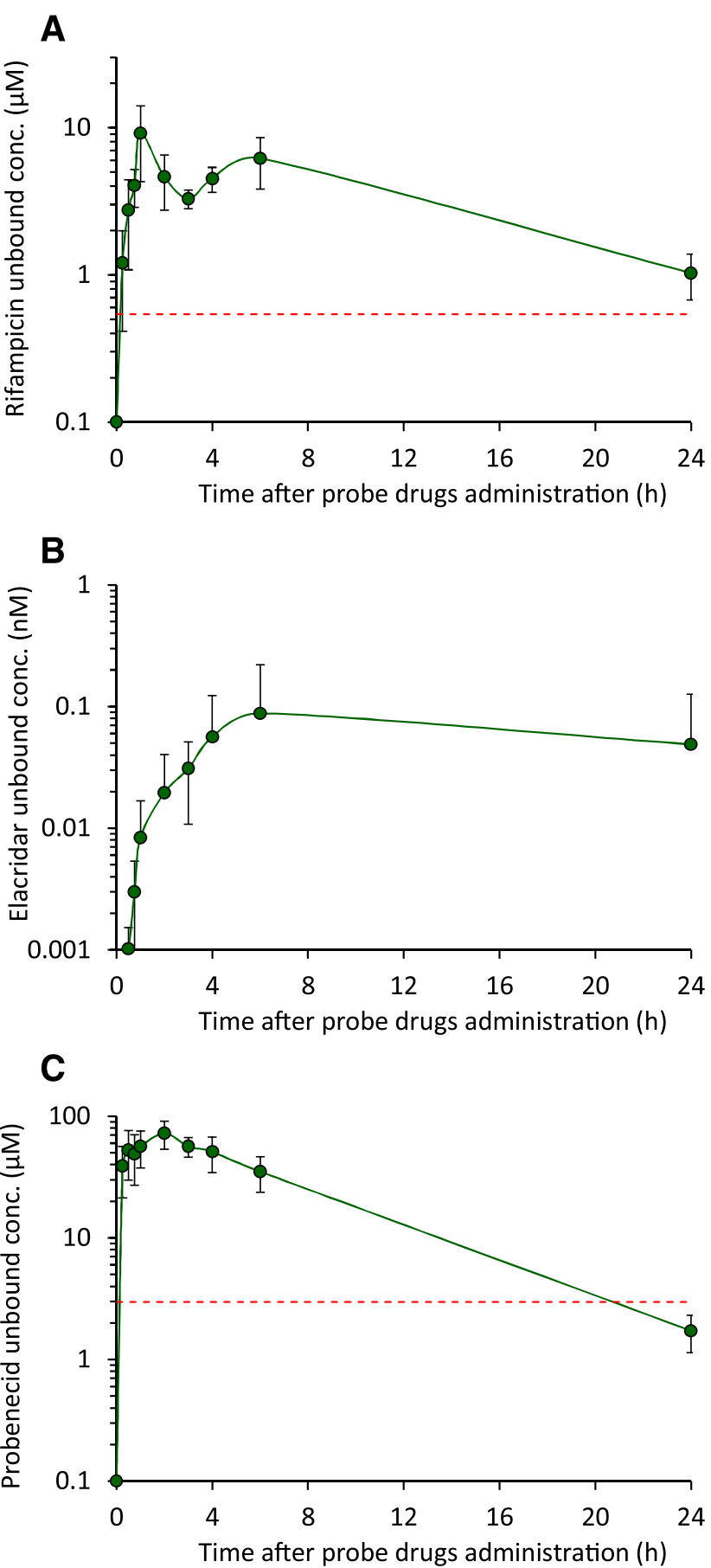

Fig. 3. Unbound plasma concentration-time profiles of inhibitor drugs dosed with probe drug cassette in cynomolgus monkey. (A) rifampicin, (B) elacridar, and (C) probenecid were dosed at $30 \mathrm{mg} / \mathrm{kg}$ each. $N=4$ (mean \pm S.D.). Horizontal lines depict hepatic uptake inhibition $\mathrm{IC}_{50}$ of rifampicin and renal OAT3 $\mathrm{IC}_{50}$ of probenecid.

coadministered with BCRP and P-gp inhibitors, respectively (Gramatté et al., 1996; Gramatté and Oertel, 1999). Although sulfasalazine is a preferred drug to probe BCRP inhibition in clinical studies (Lee et al., 2015), digoxin is commonly used to assess intestinal P-gp activity (Fenner et al., 2009; http://www.fda.gov/downloads/drugs/guidancecomplianceregulatoryinformation/guidances/ucm292362.pdf). In our preliminary studies, a combination of single-dose digoxin $(0.25 \mathrm{mg} / \mathrm{kg})$ and sulfasalazine $(1 \mathrm{mg} / \mathrm{kg})$ caused minor adverse events in the cynomolgus monkeys (i.e., emesis in the first 24 hours and lack of
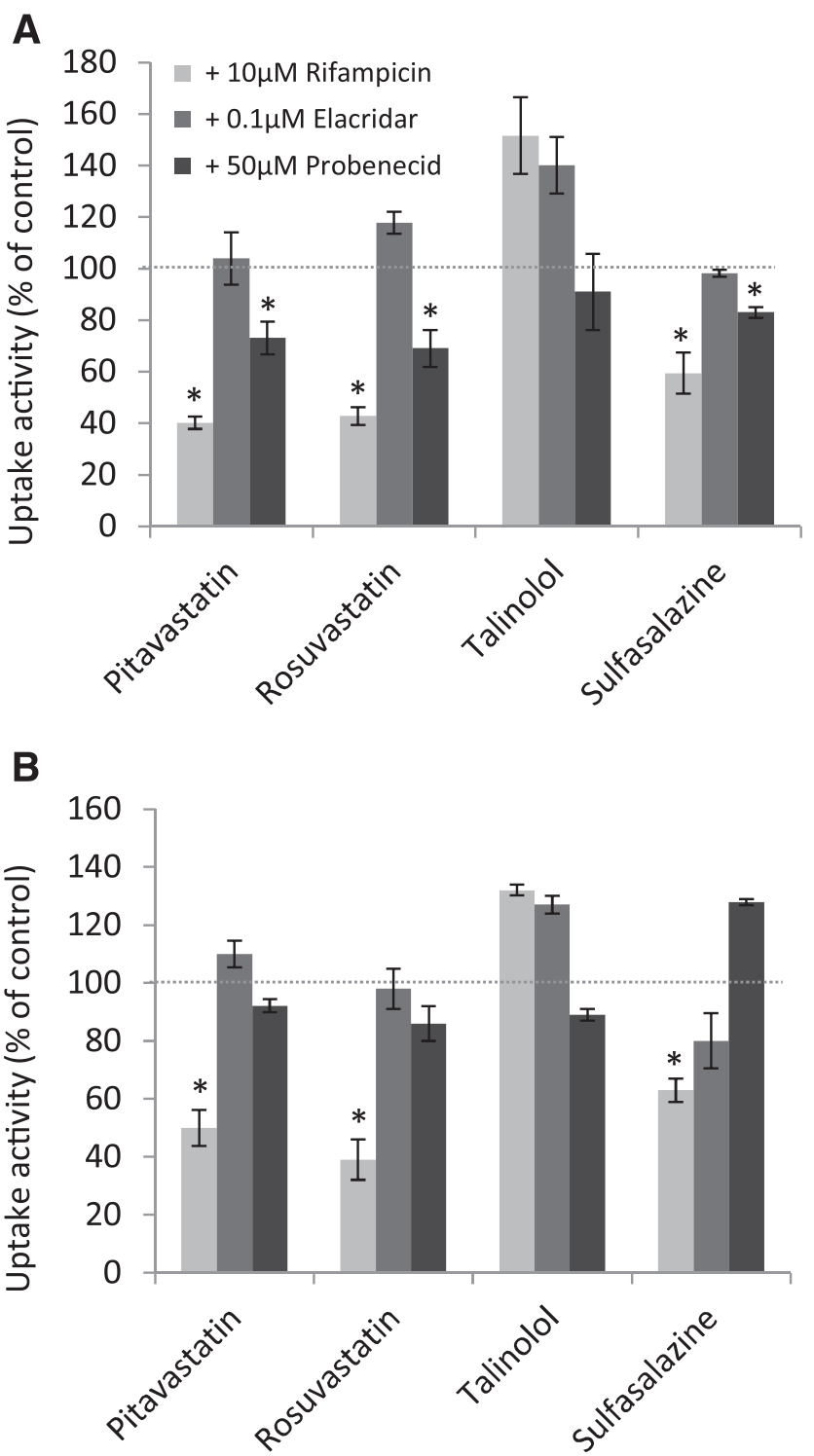

Fig. 4. In vitro uptake transport of probe substrates by plated monkey hepatocytes (A) and human hepatocytes (B). Uptake was measured in the absence and presence of rifampicin, elacridar, and probenecid at concentrations close to their unbound $\mathrm{C}_{\max }$ observed in the in vivo studies. Substrate concentration is $1 \mu \mathrm{M}$. Mean \pm S.D. $(n=3) . * P<0.05$ significantly lower than control (one-way analysis of variance).

appetite for up to 96 hours) (data not shown). We speculated that digoxin is the major source for these effects on the basis of its toxicity profile in humans (MacLeod-Glover et al., 2016), and thus considered talinolol as an alternative to measure P-gp function. Talinolol is very similar to digoxin in regard to the disposition attributes in human. For instance, P-gp inhibitors such as curcumin, erythromycin, and verapamil increase oral exposure of both drugs in humans (Gramatté et al., 1996; Gramatté and Oertel, 1999; Fenner et al., 2009). Additionally, clinical pharmacokinetics of both talinolol and digoxin are influenced by rifampicin-induced P-gp-mediated secretion (Greiner et al., 1999; Westphal et al., 2000). Finally, the four probe drugs are minimally metabolized in the human and monkey (Elsby et al., 2012; Shitara et al., 2013; Takahashi et al., 2013; Karibe et al., 2015, 2018; El-Kattan et al., 2016; Shen et al., 2016), making them suitable candidates to reliably probe transporter activity.

Of the transporter-mediated clinical DDIs, interactions involving OATP1B1/1B3 often result in larger exposure change for victim drugs 
CynoBCRP mediated transport

A

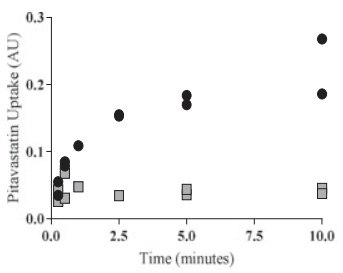

CynoP-gp mediated transport

E

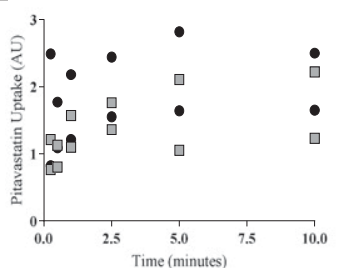

B

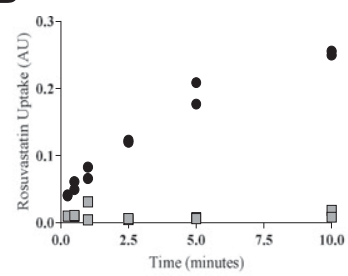

$\mathbf{F}$

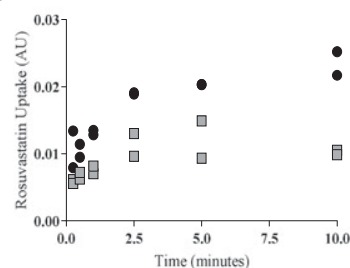

C

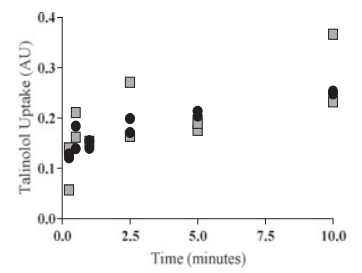

G

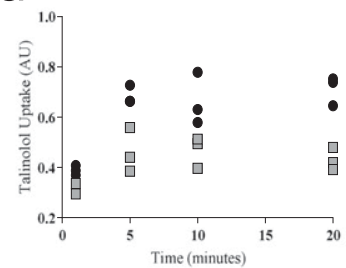

D

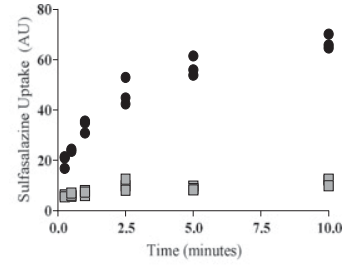

H

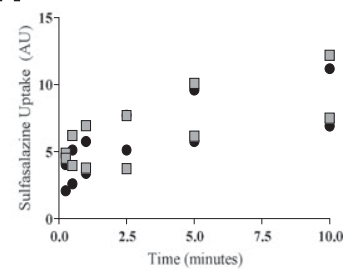

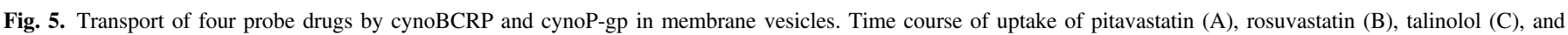

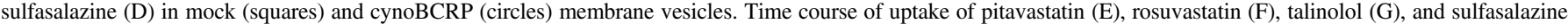
(H) in mock (squares) and cynoP-gp (circles) membrane vesicles $(n=2-3)$.

and are of particular interest for risk mitigation during drug design (Giacomini et al., 2010; Varma et al., 2017a; El-Kattan and Varma, 2018). Rifampicin dramatically increased exposure of OATP substrates pitavastatin and rosuvastatin in cynomolgus monkey (Figs. 1 and 2). Given high oral absorption (60\%-80\%) (Ufuk et al., 2018) and negligible renal clearance in monkey, pitavastatin interaction may predominantly involve OATP1B1/1B3. Rosuvastatin interaction is most likely driven by multiple mechanisms (i.e., intestinal efflux and hepatic uptake), and thus, could serve to confirm the interactions with pitavastatin (OATP1B) and sulfasalazine (BCRP). Moreover, change in rosuvastatin renal clearance can be used as a surrogate for OAT3 inhibition. However, oral AUC change of about 39- and 21-fold for pitavastatin and rosuvastatin observed in this study in the monkeys is much higher than that noted in humans ( 3- to sevenfold for both statins) (Prueksaritanont et al., 2014, 2017). Although the magnitude change is also considerably higher than reported by others at slightly lower doses of rifampicin in cynomolgus monkey (up to $15 \mathrm{mg} / \mathrm{kg}$ ) (Shen et al., 2013; Watanabe et al., 2015), the current results are nevertheless in line with our previous report in which a single oral rifampicin dose $(30 \mathrm{mg} / \mathrm{kg})$ increased AUC of oral pitavastatin and rosuvastatin by $\sim 19$ - and $\sim 15$-fold, respectively (Ufuk et al., 2018). Rifampicin-unbound $\mathrm{C}_{\max }$ achieved in monkey $(\sim 10 \mu \mathrm{M})$ at $30 \mathrm{mg} / \mathrm{kg}$ dose is relatively higher ( $\sim 2$ - to fivefold) than the unbound $C_{\max }$ in human ( 2-6 $\mu \mathrm{M}$ ) following single $600 \mathrm{mg}$ dose (Varma et al., 2012; Prueksaritanont et al., 2014; Yoshikado et al., 2017). Although this may contribute to the higher exposure change in the monkey study to some degree, other factors such as involvement of OATP2B1 in hepatic uptake clearance in humans-which is not inhibitable by rifampicin at in vivo relevant plasma concentrations (OATP2B1 in vitro $\mathrm{IC}_{50}>$ $60 \mu \mathrm{M})$ - may also contribute to difference in AUC ratios of statins. Notably, monkeys express low levels of OATP2B1 in the liver, suggesting that the fraction transported by OATP1B1/1B3 is relatively higher in monkeys than in humans (Wang et al., 2015). In contrast, magnitude of interactions registered in this study with the probe substrates and BCRP/P-gp inhibitor (elacridar) is comparable with previous reports (Karibe et al., 2015, 2018). Although clinical data are limited, the AUC change for sulfasalazine with a BCRP inhibitor (curcumin) is about threefold in humans (Kusuhara et al., 2012), which is comparable to its exposure change noted in the monkey. Collectively, the mechanisms of transporter interactions are well preserved across the two species. However, further diligence via in vitro studies and
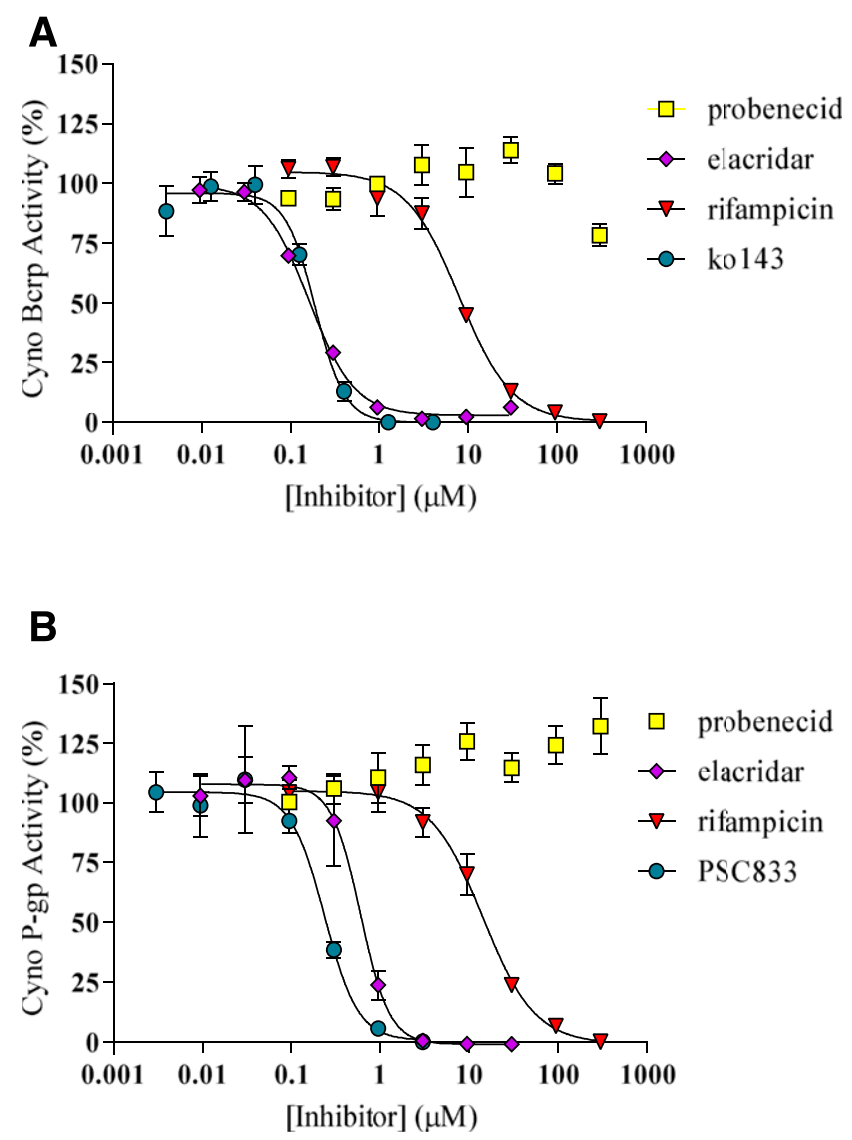

Fig. 6. Inhibition of cynoBCRP-mediated rosuvastatin transport (A) and cynoP-gpmediated uptake of $\mathrm{N}$-methyl quinidine transport (B) by rifampicin, elacridar, probenecid, and inhibitor controls in membrane vesicles. 
TABLE 5

In vitro and in vivo inhibition potency of intestinal efflux transporters by inhibitors

\begin{tabular}{|c|c|c|c|c|c|c|c|c|c|c|}
\hline Inhibitors & $\begin{array}{l}\text { Cyno P-gp } \\
\mathrm{IC}_{50}(\mu \mathrm{M})\end{array}$ & $\begin{array}{c}\text { Cyno BCRP } \\
\text { IC }_{50}(\mu \mathrm{M})\end{array}$ & $\begin{array}{l}\text { Human P-gp } \\
\mathrm{IC}_{50}(\mu \mathrm{M})^{a}\end{array}$ & $\begin{array}{l}\text { Human BCRP } \\
\text { IC }_{50}(\mu \mathrm{M})^{a}\end{array}$ & $\begin{array}{l}\text { Cyno Gut } \\
\text { conc }(\mu \mathrm{M})^{b}\end{array}$ & $\begin{array}{l}\text { Human Gut } \\
\text { conc }(\mu \mathrm{M})^{b}\end{array}$ & $\begin{array}{c}\text { Cyno P-gp } \\
\mathrm{I}_{\text {Gut }} / \mathrm{IC}_{50}\end{array}$ & $\begin{array}{c}\text { Cyno BCRP } \\
\mathrm{I}_{\mathrm{Gut}} / \mathrm{IC}_{50}\end{array}$ & $\begin{array}{l}\text { Human P-gp } \\
\mathrm{I}_{\mathrm{Gut}} / \mathrm{IC}_{50}\end{array}$ & $\begin{array}{c}\text { Human BCRP } \\
\mathrm{I}_{\mathrm{Gu}} / \mathrm{IC}_{50}\end{array}$ \\
\hline & 14.3 & 79 & 23 & 14 & 3645 & 2916 & 255 & 46 & 127 & 208 \\
\hline Elacridar & 0.6 & 0.16 & 0.09 & 0.5 & 5319 & 2837 & 8865 & 33,245 & 31,521 & 5674 \\
\hline Probenecid & - & - & - & - & - & - & - & - & - & - \\
\hline $\begin{array}{l}\text { PSC833 (P-gp } \\
\text { control) }\end{array}$ & 0.24 & - & 0.03 & - & - & - & - & - & - & - \\
\hline $\begin{array}{l}\text { Ko143 (BCRP } \\
\text { control) }\end{array}$ & - & 0.19 & - & 1 & - & - & - & - & - & - \\
\hline
\end{tabular}

conc, concentration.

${ }^{a}$ Human $\mathrm{IC}_{50}$ values were extracted from University of Washington drug interaction database (www.druginteractioninfo.org).

${ }^{b}$ Monkey $\mathrm{I}_{\text {Gut }}$ was calculated based on rifampicin/elacridar dose of $30 \mathrm{mg} / \mathrm{kg}$ and assuming luminal volume of $10 \mathrm{ml} / \mathrm{kg}$. Human $\mathrm{I}_{\mathrm{Gut}}$ was calculated based on rifampicin/elacridar dose of $600 /$ $400 \mathrm{mg}$ and assuming luminal volume of $250 \mathrm{ml}$ (Amidon et al., 1995; Kararli, 1995).

(一), not calculated.

mechanistic modeling would be needed to quantitatively extrapolate monkey DDIs to human.

Interestingly, probenecid significantly inhibited statin uptake by monkey hepatocytes (Fig. 4A), which may explain $\sim 2$ - to threefold change in plasma exposure in our monkey studies (Tables 1 and 2). However, the effect of probenecid on uptake is nominal and not statistically significant in human hepatocytes (Fig. 4B). Previous studies have shown in vitro OATP1B1 inhibition by probenecid at clinically relevant concentrations (Izumi et al., 2016). Clinical data on the inhibitory effect of probenecid on hepatic uptake are not directly available. However, we believe that $\sim 50 \%-70 \%$ increase in fexofenadine plasma AUC by probenecid is at least partly due to hepatic uptake inhibition (Liu et al., 2008). Fexofenadine bioavailability is 35\% (Lappin et al., 2010), and therefore, inhibition of OAT3-mediated renal clearance resulting in a change in the amount of dose excreted in urine from $\sim 8 \%$ to $4 \%$ alone cannot explain the noted AUC change (Liu et al., 2008). In contrast, sulfasalazine uptake in monkey and human hepatocytes was inhibited by rifampicin in vitro; therefore, contribution of uptake clearance to sulfasalazine AUC change when dosed with rifampicin cannot be ruled out. Further studies are warranted in understanding the role of hepatic uptake in the disposition of sulfasalazine, which is currently recommended as a clinical probe to assess BCRP inhibition.

In our previous study, the AUC ratios of both i.v. and oral pitavastatin with increasing doses of oral rifampicin $(1-30 \mathrm{mg} / \mathrm{kg}$ ) in cynomolgus monkey were well predicted from in vitro $\mathrm{IC}_{50}$ data, assuming inhibition of only OATP1B-mediated hepatic uptake (Ufuk et al., 2018). However, in case of rosuvastatin, AUC ratios were well predicted following i.v. administration of rosuvastatin, but underpredicted following oral dose, particularly at higher doses of rifampicin. The current study suggests that the disconnect in the in vitro-in vivo extrapolation of rosuvastatinrifampicin DDIs is due to lack of consideration to BCRP-mediated intestinal efflux. Rifampicin increased plasma exposure of BCRP (sulfasalazine) and P-gp (talinolol) substrates, implying that rifampicin inhibited intestinal efflux in cynomolgus monkey. Rifampicin inhibited cynoBCRP and cynoP-gp in our in vitro studies using membrane vesicles (Fig. 6). Simple $\mathrm{I}_{\mathrm{Gut}} / \mathrm{IC}_{50}$ calculations suggested that rifampicin considerably inhibits intestinal efflux at the dose employed (Table 5). A comparison of these findings with $\mathrm{IC}_{50}$ for human BCRP and P-gp and the corresponding $\mathrm{I}_{\mathrm{Gut}} / \mathrm{IC}_{50}$ values indicates that rifampicin can impact clinical pharmacokinetics of BCRP and P-gp substrates at the dose (600 mg single dose) recommended to probe OATP1B activity. Similarly, $\mathrm{I}_{\max , \mathrm{u}} / \mathrm{IC}_{50}$ values of $\sim 0.14-0.76$ imply potential for rifampicin to inhibit P-gp- and BCRP-mediated biliary secretion in both species. These values are likely an underestimation given rifampicin shows high liver-to-plasma-free concentrations in vivo (Zaher et al., 2008). However, contribution of this mechanism to the observed plasma exposure changes of probe substrates depends on multiple components of extended clearance model (Varma et al., 2014). This in vitro-in vivo analysis collectively supports the premise that rifampicin clinical DDIs should be rationalized by accounting for both OATP1B-mediated hepatic uptake and biliary efflux, as well as intestinal efflux of victim drugs.

In agreement with others (Tahara et al., 2006; Shen et al., 2013, 2016; Karibe et al., 2015), the results of the present study support the utility of the cynomolgus monkey as a model species for assessing transportermediated DDIs involving OATPs, P-gp, BCRP, and OAT1/3. In the case of OATPs and OAT1/3, efforts have been made to identify, characterize, and validate suitable biomarkers to support clinical DDI studies (Lai et al., 2016; Rodrigues et al., 2018). Therefore, it may be possible to use the cynomolgus monkey, integrated with clinically validated biomarkers, to quickly discharge DDI risk or prioritize dedicated (drug probe-based) clinical DDI studies. To date, however, suitable biomarkers for intestinal P-gp and BCRP have not been identified, and DDI predictions as well as in vitro-in vivo extrapolations involving both transporters remain challenging. In this regard, the cynomolgus monkey has potential to serve as a model to drive in vitro-in vivo extrapolations involving P-gp and BCRP inhibition and support human DDI risk assessment. Obviously, one would have to consider, and account for, species differences in perpetrator absorption, exposure, and intrinsic inhibitory potency. As described in this work, the latter can be addressed with in vitro studies using human and monkey vesicles and primary hepatocytes.

Although DDIs involving hepatic OCT1 and renal OCT2/MATEs are also suggested to be clinically relevant, the change in pharmacokinetics of substrate drugs is often marginal (AUC ratio <1.5) (Varma et al., 2017a). Arguably, preclinical DDI risk assessments in such cases do not definitively inform the decision to progress NMEs to clinical development. Therefore, the proposed cocktail did not include OCT1/OCT2/ MATE probe substrates. Nevertheless, a separate DDI study in cynomolgus monkey using metformin as a probe substrate could be conducted (Shen et al., 2016). Alternatively, changes in creatinine levels measured in preclinical toxicology studies potentially indicate inhibition of OCT2 and/or MATEs (Fuchs and Hewitt, 2011). Such findings can form the basis for monitoring endogenous biomarkers such as creatinine and $\mathrm{N}^{1}$-methylnicotinamide in first-in-man studies, which in turn can determine the need for a dedicated clinical DDI study with a drug probe (Rodrigues et al., 2018).

\section{Conclusion}

In conclusion, the proposed approach for simultaneously assessing major transporter-mediated DDIs using a probe substrate cocktail in cynomolgus monkey can be valuable in drug design and clinical 
development. Such preclinical in vivo data in conjunction with in vitro interaction assessments can help flag the unforeseen interaction risks or avoid unnecessary clinical DDI studies. Finally, this study confirms similarities in drug handling by major transporters between human and cynomolgus monkey, suggesting the latter is a viable in vivo model for mechanistic investigation of transporter-mediated disposition and pharmacokinetic interaction.

\section{Acknowledgments}

We especially thank Solvo Biotechnology (Budapest, Hungary) for providing cynomolgus monkey BCRP and P-gp membrane vesicles. We acknowledge Marko Andric, Emese Kis, and Beáta Tóth (Solvo Biotechnology) for the technical inputs regarding the membrane vesicle assays.

\section{Authorship Contributions}

Participated in research design: Kosa, Lazzaro, Rodrigues, Tremaine, Varma.

Conducted experiments: Kosa, Lazzaro, Bi, Tierney, Gates, Modi.

Performed data analysis: Kosa, Lazzaro, Bi, Tierney, Gates, Modi, Costales, Varma.

Wrote or contributed to the writing of the manuscript: Kosa, Lazzaro, Bi, Tierney, Gates, Modi, Costales, Rodrigues, Tremaine, Varma.

\section{References}

Amidon GL, Lennernäs H, Shah VP, and Crison JR (1995) A theoretical basis for a biopharmaceutic drug classification: the correlation of in vitro drug product dissolution and in vivo bioavailability. Pharm Res 12:413-420.

Amundsen R, Christensen H, Zabihyan B, and Åsberg A (2010) Cyclosporine A, but not tacrolimus, shows relevant inhibition of organic anion-transporting protein 1B1-mediated transport of atorvastatin. Drug Metab Dispos 38:1499-1504

Begley DJ (2004) ABC transporters and the blood-brain barrier. Curr Pharm Des 10:1295-1312. Bentz J, O'Connor MP, Bednarczyk D, Coleman J, Lee C, Palm J, Pak YA, Perloff ES, Reyner E, Balimane P, et al. (2013) Variability in P-glycoprotein inhibitory potency (IC50) using various in vitro experimental systems: implications for universal digoxin drug-drug interaction risk assessment decision criteria. Drug Metab Dispos 41:1347-1366.

Bi YA, Lin J, Mathialagan S, Tylaska L, Callegari E, Rodrigues AD, and Varma MVS (2018) Role of hepatic organic anion transporter 2 in the pharmacokinetics of R- and S-warfarin: in vitro studies and mechanistic evaluation. Mol Pharm 15:1284-1295.

Bi YA, Scialis RJ, Lazzaro S, Mathialagan S, Kimoto E, Keefer J, Zhang H, Vildhede AM, Costales C, Rodrigues AD, et al. (2017) Reliable rate measurements for active and passive hepatic uptake using plated human hepatocytes. AAPS $J$ 19:787-796.

Bjornsson TD, Callaghan JT, Einolf HJ, Fischer V, Gan L, Grimm S, Kao J, King SP, Miwa G, Ni L, et al.; Pharmaceutical Research and Manufacturers of America (PhRMA) Drug Metabolism/ Clinical Pharmacology Technical Working Group; FDA Center for Drug Evaluation and Research (CDER) (2003) The conduct of in vitro and in vivo drug-drug interaction studies: a Pharmaceutical Research and Manufacturers of America (PhRMA) perspective. Drug Metab Dispos 31:815-832.

Bloomer J, Derimanov G, Dumont E, Ellens H, and Matheny C (2013) Optimizing the in vitro and clinical assessment of drug interaction risk by understanding co-medications in patient populations. Expert Opin Drug Metab Toxicol 9:737-751.

de Bruyn T, Ufuk A, Cantrill C, Kosa RE, Bi YA, Niosi M, Modi S, Rodrigues AD, Tremaine LM, Varma MV, et al. (2018) Predicting human clearance of OATP substrates using cynomolgus monkey: in vitro-in vivo scaling of hepatic uptake clearance. Drug Metab Dispos DOI: 10.1124/ dmd.118.081315 [published ahead of print].

El-Kattan AF, Varma MV, Steyn SJ, Scott DO, Maurer TS, and Bergman A (2016) Projecting ADME behavior and drug-drug interactions in early discovery and development: application of the extended clearance classification system. Pharm Res 33:3021-3030.

El-Kattan AF and Varma MVS (2018) Navigating transporter sciences in pharmacokinetics characterization using the extended clearance classification system. Drug Metab Dispos 46:729-739.

Elsby R, Hilgendorf C, and Fenner K (2012) Understanding the critical disposition pathways of statins to assess drug-drug interaction risk during drug development: it's not just about OATP1B1. Clin Pharmacol Ther 92:584-598.

Feng B, LaPerle JL, Chang G, and Varma MV (2010) Renal clearance in drug discovery and development: molecular descriptors, drug transporters and disease state. Expert Opin Drug Metab Toxicol 6:939-952.

Fenner KS, Troutman MD, Kempshall S, Cook JA, Ware JA, Smith DA, and Lee CA (2009) Drugdrug interactions mediated through P-glycoprotein: clinical relevance and in vitro-in vivo correlation using digoxin as a probe drug. Clin Pharmacol Ther 85:173-181.

Fuchs TC and Hewitt P (2011) Biomarkers for drug-induced renal damage and nephrotoxicity: an overview for applied toxicology. AAPS $J$ 13:615-631.

Gertz M, Cartwright CM, Hobbs MJ, Kenworthy KE, Rowland M, Houston JB, and Galetin A (2013) Cyclosporine inhibition of hepatic and intestinal CYP3A4, uptake and efflux transporters: application of PBPK modeling in the assessment of drug-drug interaction potential. Pharm Res 30:761-780

Giacomini KM, Huang SM, Tweedie DJ, Benet LZ, Brouwer KL, Chu X, Dahlin A, Evers R, Fischer V, Hillgren KM, et al.; International Transporter Consortium (2010) Membrane transporters in drug development. Nat Rev Drug Discov 9:215-236.

Gramatté T and Oertel R (1999) Intestinal secretion of intravenous talinolol is inhibited by luminal R-verapamil. Clin Pharmacol Ther 66:239-245.
Gramatté T, Oertel R, Terhaag B, and Kirch W (1996) Direct demonstration of small intestinal secretion and site-dependent absorption of the beta-blocker talinolol in humans. Clin Pharmaco Ther 59:541-549.

Greiner B, Eichelbaum M, Fritz P, Kreichgauer H-P, von Richter O, Zundler J, and Kroemer HK (1999) The role of intestinal P-glycoprotein in the interaction of digoxin and rifampin. J Clin Invest 104:147-153.

Izumi S, Nozaki Y, Komori T, Maeda K, Takenaka O, Kusano K, Yoshimura T, Kusuhara H, and Sugiyama Y (2013) Substrate-dependent inhibition of organic anion transporting polypeptide 1B1: comparative analysis with prototypical probe substrates estradiol-17 $\beta$-glucuronide, estrone-3-sulfate, and sulfobromophthalein. Drug Metab Dispos 41:1859-1866.

Izumi S, Nozaki Y, Komori T, Takenaka O, Maeda K, Kusuhara H, and Sugiyama Y (2016) Investigation of fluorescein derivatives as substrates of organic anion transporting polypeptide (OATP) 1B1 to develop sensitive fluorescence-based OATP1B1 inhibition assays. Mol Pharm 13:438-448.

Kararli TT (1995) Comparison of the gastrointestinal anatomy, physiology, and biochemistry of humans and commonly used laboratory animals. Biopharm Drug Dispos 16:351-380.

Karibe T, Hagihara-Nakagomi R, Abe K, Imaoka T, Mikkaichi T, Yasuda S, Hirouchi M, Watanabe N, Okudaira N, and Izumi T (2015) Evaluation of the usefulness of breast cancer resistance protein (BCRP) knockout mice and BCRP inhibitor-treated monkeys to estimate the clinical impact of BCRP modulation on the pharmacokinetics of BCRP substrates. Pharm Res 32:1634-1647.

Karibe T, Imaoka T, Abe K, and Ando O (2018) Curcumin as an in vivo selective intestinal breas cancer resistance protein inhibitor in cynomolgus monkeys. Drug Metab Dispos 46:667-679.

Koepsell H, Lips K, and Volk C (2007) Polyspecific organic cation transporters: structure, function, physiological roles, and biopharmaceutical implications. Pharm Res 24:1227-1251.

Kusuhara H, Furuie H, Inano A, Sunagawa A, Yamada S, Wu C, Fukizawa S, Morimoto N, Ieiri I, Morishita M, et al. (2012) Pharmacokinetic interaction study of sulphasalazine in healthy subjects and the impact of curcumin as an in vivo inhibitor of BCRP. Br J Pharmacol 166 $1793-1803$.

Lai Y, Mandlekar S, Shen H, Holenarsipur VK, Langish R, Rajanna P, Murugesan S, Gaud N, Selvam S, Date O, et al. (2016) Coproporphyrins in plasma and urine can be appropriate clinical biomarkers to recapitulate drug-drug interactions mediated by organic anion transporting polypeptide inhibition. J Pharmacol Exp Ther 358:397-404.

Lappin G, Shishikura Y, Jochemsen R, Weaver RJ, Gesson C, Houston B, Oosterhuis B, Bjerrum OJ, Rowland M, and Garner C (2010) Pharmacokinetics of fexofenadine: evaluation of a microdose and assessment of absolute oral bioavailability. Eur J Pharm Sci 40:125-131.

Lee CA, O'Connor MA, Ritchie TK, Galetin A, Cook JA, Ragueneau-Majlessi I, Ellens H, Feng B, Taub ME, Paine MF, et al. (2015) Breast cancer resistance protein (ABCG2) in clinical pharmacokinetics and drug interactions: practical recommendations for clinical victim and perpetrator drug-drug interaction study design. Drug Metab Dispos 43:490-509.

Lee W and Kim RB (2004) Transporters and renal drug elimination. Annи Rev Pharmacol Toxicol 44:137-166.

Li R, Barton HA, and Varma MV (2014) Prediction of pharmacokinetics and drug-drug interactions when hepatic transporters are involved. Clin Pharmacokinet 53:659-678.

Liu S, Beringer PM, Hidayat L, Rao AP, Louie S, Burckart GJ, and Shapiro B (2008) Probenecid, but not cystic fibrosis, alters the total and renal clearance of fexofenadine. J Clin Pharmacol $\mathbf{4 8}$ 957-965.

MacLeod-Glover N, Mink M, Yarema M, and Chuang R (2016) Digoxin toxicity: case for retiring its use in elderly patients? Can Fam Physician 62:223-228

Mathialagan S, Piotrowski MA, Tess DA, Feng B, Litchfield J, and Varma MV (2017) Quantitative prediction of human renal clearance and drug-drug interactions of organic anion transporter substrates using in vitro transport data: a relative activity factor approach. Drug Metab Dispos 45:409-417.

Noé J, Portmann R, Brun ME, and Funk C (2007) Substrate-dependent drug-drug interactions between gemfibrozil, fluvastatin and other organic anion-transporting peptide (OATP) substrates on OATP1B1, OATP2B1, and OATP1B3. Drug Metab Dispos 35:1308-1314.

Prueksaritanont T, Chu X, Evers R, Klopfer SO, Caro L, Kothare PA, Dempsey C, Rasmussen S, Houle R, Chan G, et al. (2014) Pitavastatin is a more sensitive and selective organic aniontransporting polypeptide 1B clinical probe than rosuvastatin. Br J Clin Pharmacol 78:587-598

Prueksaritanont T, Tatosian DA, Chu X, Railkar R, Evers R, Chavez-Eng C, Lutz R, Zeng W, Yabut J, Chan GH, et al. (2017) Validation of a microdose probe drug cocktail for clinical drug interaction assessments for drug transporters and CYP3A. Clin Pharmacol Ther 101:519-530.

Rodrigues AD, Taskar KS, Kusuhara H, and Sugiyama Y (2018) Endogenous probes for drug transporters: balancing vision with reality. Clin Pharmacol Ther 103:434-448.

Saito Y, Maekawa K, and Ohno Y (2014) Novel draft guideline for drug interaction studies in the drug development and labeling recommendations. Regul Sci Med Prod 4:249-255.

Sawicki E, Verheijen RB, Huitema AD, van Tellingen O, Schellens JH, Nuijen B, Beijnen JH, and Steeghs N (2017) Clinical pharmacokinetics of an amorphous solid dispersion tablet of elacridar. Drug Deliv Transl Res 7:125-131.

Shen H, Liu T, Jiang H, Titsch C, Taylor K, Kandoussi H, Qiu X, Chen C, Sukrutharaj S, Kuit K, et al. (2016) Cynomolgus monkey as a clinically relevant model to study transport involving renal organic cation transporters: in vitro and in vivo evaluation. Drug Metab Dispos 44 238-249.

Shen H, Su H, Liu T, Yao M, Mintier G, Li L, Fancher RM, Iyer R, Marathe P, Lai Y, et al. (2015) Evaluation of rosuvastatin as an organic anion transporting polypeptide (OATP) probe substrate: in vitro transport and in vivo disposition in cynomolgus monkeys. J Pharmacol Exp Ther 353: 380-391.

Shen H, Yang Z, Mintier G, Han Y-H, Chen C, Balimane P, Jemal M, Zhao W, Zhang R, Kallipatti $\mathrm{S}$, et al. (2013) Cynomolgus monkey as a potential model to assess drug interactions involving hepatic organic anion transporting polypeptides: in vitro, in vivo, and in vitro-to-in vivo extrapolation. J Pharmacol Exp Ther 344:673-685.

Shitara Y, Hirano M, Sato H, and Sugiyama Y (2004) Gemfibrozil and its glucuronide inhibit the organic anion transporting polypeptide 2 (OATP2/OATP1B1:SLC21A6)-mediated hepatic uptake and CYP2C8-mediated metabolism of cerivastatin: analysis of the mechanism of the clinically relevant drug-drug interaction between cerivastatin and gemfibrozil. J Pharmacol Exp Ther 311:228-236.

Shitara Y, Horie T, and Sugiyama Y (2006) Transporters as a determinant of drug clearance and tissue distribution. Eur J Pharm Sci 27:425-446.

Shitara Y, Maeda K, Ikejiri K, Yoshida K, Horie T, and Sugiyama Y (2013) Clinical significance of organic anion transporting polypeptides (OATPs) in drug disposition: their roles in hepatic clearance and intestinal absorption. Biopharm Drug Dispos 34:45-78. 
Stopfer P, Giessmann T, Hohl K, Sharma A, Ishiguro N, Taub ME, Zimdahl-Gelling H, Gansser D, Wein M, Ebner T, et al. (2016) Pharmacokinetic evaluation of a drug transporter cocktail consisting of digoxin, furosemide, metformin, and rosuvastatin. Clin Pharmacol Ther 100 259-267.

Tahara H, Kusuhara H, Chida M, Fuse E, and Sugiyama Y (2006) Is the monkey an appropriate animal model to examine drug-drug interactions involving renal clearance? Effect of probenecid on the renal elimination of $\mathrm{H} 2$ receptor antagonists. J Pharmacol Exp Ther 316 $1187-1194$

Takahashi T, Ohtsuka T, Yoshikawa T, Tatekawa I, Uno Y, Utoh M, Yamazaki H, and Kume T (2013) Pitavastatin as an in vivo probe for studying hepatic organic anion transporting polypeptide-mediated drug-drug interactions in cynomolgus monkeys. Drug Metab Dispos 41 $1875-1882$.

Ufuk A, Kosa RE, Gao H, Bi YA, Modi S, Gates D, Rodrigues AD, Tremaine LM, Varma MVS, Houston JB, et al. (2018) In vitro-in vivo extrapolation of OATP1B-mediated drug-drug interactions in cynomolgus monkey. J Pharmacol Exp Ther 365:688-699.

Vaidyanathan J, Yoshida K, Arya V, and Zhang L (2016) Comparing various in vitro prediction criteria to assess the potential of a new molecular entity to inhibit organic anion transporting polypeptide 1B1. J Clin Pharmacol 56 (Suppl 7):S59-S72.

Varma MV, Ambler CM, Ullah M, Rotter CJ, Sun H, Litchfield J, Fenner KS, and El-Kattan AF (2010) Targeting intestinal transporters for optimizing oral drug absorption. Curr Drug Metab 11:730-742.

Varma MV, Bi YA, Kimoto E, and Lin J (2014) Quantitative prediction of transporter- and enzyme-mediated clinical drug-drug interactions of organic anion-transporting polypeptide 1B1 substrates using a mechanistic net-effect model. J Pharmacol Exp Ther 351:214-223.

Varma MV, El-Kattan AF, Feng B, Steyn SJ, Maurer TS, Scott DO, Rodrigues AD, and Tremaine LM (2017a) Extended clearance classification system (ECCS) informed approach for evaluating investigational drugs as substrates of drug transporters. Clin Pharmacol Ther 102:33-36.

Varma MV, Kimoto E, Scialis R, Bi Y, Lin J, Eng H, Kalgutkar AS, El-Kattan AF, Rodrigues AD, and Tremaine LM (2017b) Transporter-mediated hepatic uptake plays an important role in the pharmacokinetics and drug-drug interactions of montelukast. Clin Pharmacol Ther 101 406-415.

Varma MV, Lai Y, Feng B, Litchfield J, Goosen TC, and Bergman A (2012) Physiologically based modeling of pravastatin transporter-mediated hepatobiliary disposition and drug-drug interactions. Pharm Res 29:2860-2873.
Varma MV, Lin J, Bi YA, Kimoto E, and Rodrigues AD (2015a) Quantitative rationalization of gemfibrozil drug interactions: consideration of transporters-enzyme interplay and the role of circulating metabolite gemfibrozil 1-O- $\beta$-glucuronide. Drug Metab Dispos 43:1108-1118.

Varma MV, Steyn SJ, Allerton C, and El-Kattan AF (2015b) Predicting clearance mechanism in drug discovery: extended clearance classification system (ECCS). Pharm Res 32:3785-3802.

Wang L, Prasad B, Salphati L, Chu X, Gupta A, Hop CE, Evers R, and Unadkat JD (2015) Interspecies variability in expression of hepatobiliary transporters across human, dog, monkey, and rat as determined by quantitative proteomics. Drug Metab Dispos 43:367-374.

Watanabe M, Watanabe T, Yabuki M, and Tamai I (2015) Dehydroepiandrosterone sulfate, a useful endogenous probe for evaluation of drug-drug interaction on hepatic organic anion transporting polypeptide (OATP) in cynomolgus monkeys. Drug Metab Pharmacokinet 30: 198-204.

Westphal K, Weinbrenner A, Zschiesche M, Franke G, Knoke M, Oertel R, Fritz P, von Richter O, Warzok R, Hachenberg T, et al. (2000) Induction of P-glycoprotein by rifampin increases intestinal secretion of talinolol in human beings: a new type of drug/drug interaction. Clin Pharmacol Ther 68:345-355.

Yoshikado T, Maeda K, Furihata S, Terashima H, Nakayama T, Ishigame K, Tsunemoto K, Kusuhara H, Furihata KI, and Sugiyama Y (2017) A clinical cassette dosing study for evaluating the contribution of hepatic OATPs and CYP3A to drug-drug interactions. Pharm Res 34: $1570-1583$.

Zaher H, Meyer zu Schwabedissen HE, Tirona RG, Cox ML, Obert LA, Agrawal N, Palandra J, Stock JL, Kim RB, and Ware JA (2008) Targeted disruption of murine organic aniontransporting polypeptide $1 \mathrm{~b} 2$ (Oatp1b2/Slco1b2) significantly alters disposition of prototypical drug substrates pravastatin and rifampin. Mol Pharmacol 74:320-329.

Zhang H, Cui D, Wang B, Han YH, Balimane P, Yang Z, Sinz M, and Rodrigues AD (2007) Pharmacokinetic drug interactions involving 17alpha-ethinylestradiol: a new look at an old drug. Clin Pharmacokinet 46:133-157.

Address correspondence to: Dr. Manthena V. Varma, Pharmacokinetics, Dynamics, and Metabolism, Pfizer Worldwide R\&D, Pfizer, Eastern Point Road, MS 8220-2451, Groton, CT 06340. E-mail: manthena.v.varma@pfizer.com 